This item was submitted to Loughborough's Research Repository by the author.

Items in Figshare are protected by copyright, with all rights reserved, unless otherwise indicated.

\title{
Structured biodegradable polymeric microparticles for drug delivery produced using flow focusing glass microfluidic devices
}

\section{PLEASE CITE THE PUBLISHED VERSION}

http://dx.doi.org/10.1021/acsami.5b06943

\section{PUBLISHER}

(c) American Chemical Society

\section{VERSION}

AM (Accepted Manuscript)

\section{PUBLISHER STATEMENT}

This work is made available according to the conditions of the Creative Commons Attribution-NonCommercialNoDerivatives 4.0 International (CC BY-NC-ND 4.0) licence. Full details of this licence are available at: https://creativecommons.org/licenses/by-nc-nd/4.0/

\section{LICENCE}

CC BY-NC-ND 4.0

\section{REPOSITORY RECORD}

Ekanem, Ekanem E., Seyed Ali Nabavi, Goran T. Vladisavljevic, and Sai Gu. 2015. "Structured Biodegradable Polymeric Microparticles for Drug Delivery Produced Using Flow Focusing Glass Microfluidic Devices”. figshare. https://hdl.handle.net/2134/19339. 


\title{
Structured biodegradable polymeric microparticles
}

\section{for drug delivery produced using flow focusing}

\section{glass microfluidic devices}

\author{
Ekanem E. Ekanem, ${ }^{\dagger}$ Seyed Ali Nabavi, ${ }^{\ddagger}$ Goran T. Vladisavljević, ${ }^{*}{ }^{\dagger}$ and Sai Gu ${ }^{\#}$ \\ ${ }^{\dagger}$ Department of Chemical Engineering, Loughborough University, Loughborough, LE11 \\ 3TU, United Kingdom.
}

${ }^{\ddagger}$ School of Energy, Environment \& Agrifood (SEEA), Department of Offshore, Process \& Energy Engineering, Cranfield University, Cranfield, MK43 0AL, United Kingdom.

\#Department of Chemical and Process Engineering, Faculty of Engineering and Physical Sciences, University of Surrey, Guildford, GU2 7XH, United Kingdom.

${ }^{*}$ Corresponding author's address: Department of Chemical Engineering, Loughborough University, Loughborough, LE11 3TU, United Kingdom. Phone number +441509222518; fax number +441509223923; email: g.vladisavljevic@lboro.ac.uk

ABSTRACT: Biodegradable poly(DL-lactic acid) (PLA) and poly(lactic-co-glycolic acid) (PLGA) microparticles with tunable size, shape, internal structure and surface morphology were produced by counter-current flow focusing in axisymmetric (3D) glass capillary devices. The dispersed phase was composed of $0.5-2 \mathrm{wt} \%$ polymer solution in a volatile 
organic solvent (ethyl acetate or dichloromethane) and the continuous phase was $5 \mathrm{wt} \%$ aqueous poly(vinyl alcohol) solution. The droplets with a coefficient of variation in dripping regime below $2.5 \%$ were evaporated to form polymeric particles with uniform sizes ranging between 4-30 $\mu \mathrm{m}$. The particle microstructure and surface roughness were modified by adding nanofiller (montmorillonite nanoclay) or porogen (2-methylpentane) in the dispersed phase to form less porous polymer matrix or porous particles with golf-ball-like dimpled surface, respectively. The presence of $2-4 \mathrm{wt} \%$ nanoclay in the host polymer significantly reduced the release rate of paracetamol and prevented the early burst release, as a result of reduced polymer porosity and tortuous path for the diffusing drug molecules. Numerical modelling results using the volume of fluid-continuum surface force model agreed well with experimental behaviour and revealed trapping of nanoclay particles in the dispersed phase upstream of the orifice at low dispersed phase flow rates and for $4 \mathrm{wt} \%$ nanoclay content, due to vortex formation. Janus PLA/PCL (polycaprolactone) particles were produced by solvent evaporation-induced phase separation within organic phase droplets containing $3 \%(\mathrm{v} / \mathrm{v})$ PLA/PCL (30/70 or 70/30) mixture in dichloromethane. A strong preferential adsorption of Rhodamine 6G dye onto PLA was utilized to identify PLA portions of the Janus particles by Confocal Laser Scanning Microscopy (CLSM). Uniform hemispherical PCL particles were produced by dissolution of PLA domes with acetone.

KEYWORDS: Microfluidic flow focusing, Biodegradable microspheres, Drug delivery systems, Poly(lactic acid), Poly(lactic-co-glycolic acid), Nanoclay, Janus particle, Hemispherical particle. 


\section{INTRODUCTION}

Conventional oral and intravenous drug administration routes are characterized by rapid drug release and absorption. ${ }^{1}$ Limitations such as poor patient compliance (due to missing or altering dosages) and difficulty in attainment of steady state conditions (as a result of peakvalley plasma concentration fluctuations) have led to poor drug efficacy and toxicity as consequences of underdosing and overdosing of drugs respectively in patients. ${ }^{2}$ Drug delivery systems have been designed to overcome these limitations and extend, delay and target drug release $^{3-6}$. When properly designed, controlled drug delivery systems should be able to deliver drugs at a predetermined rate and manner, either locally or systemically, for a specific period of time after drug administration, which can eliminate excessive fluctuations of drug plasma concentrations. ${ }^{7}$

Biocompatible polymeric materials such as non-biodegradable hydrophobic polymers, ${ }^{8}$ hydrogels, ${ }^{9}$ water soluble polymers, ${ }^{10}$ and synthetic biodegradable polymers have been widely used as vehicles for drug delivery. ${ }^{1}$ Biodegradable polymers have been mainly used in the form of microspheres, since they offer high surface area for adhesion and drug release and a low drag force during mobility in fluids. ${ }^{11}$ The most common biodegradable synthetic polymers are poly(lactic acid) (PLA), poly(lactic-co-glycolic acid) (PLGA) ${ }^{12}$, and polycaprolactone (PCL), ${ }^{1,13}$ due to their approval by FDA (Food and Drug Administration) ${ }^{14}$ and environmentally friendly nature. In order to meet different dosage requirements, there is a strong need to modify drug solubility and release rate by incorporation of different additives into a host biodegradable polymer. 
Nanoclays are FDA approved plate-like nanoparticles of layered mineral silicates that can be integrated into the polymer matrix to improve mechanical and rheological properties of polymeric microspheres and increase the solubility and bioavailability of drugs such as ibuprofen, indomethacin, diclofenac, naproxen, fenbufen, and paracetamol. ${ }^{2}$ Nanoclays also have highly desirable dietary, bacterial and metabolic detoxification characteristics ${ }^{15}$.

Another method of modification of drug release kinetics is internal phase separation caused by addition of non-solvent in the mixture of polymer and solvent prior to emulsification. Depending on polymer to non-solvent ratio and the type of solvent, non-solvent and polymer, phase separation within emulsion droplets (triggered by solvent evaporation) can lead to core/shell, occluded, acorn or heteroaggregated particle morphology. ${ }^{16}$ Hole-shell and crescent-moon-shaped microparticles can be synthesised from acorn-shaped biphasic droplets by polymerisation of one of the phases. ${ }^{17,18}$ Such unique morphologies can modify drug release profile $e^{12,19,20}$ and increase the potential for alternative routes of drug administration.

Emulsion-solvent evaporation is one of the most common methods for fabrication of polymeric microspheres from pre-formed polymers. Emulsification can be carried out using conventional top-down methods based on breaking larger droplets into smaller ones, ${ }^{21}$ such as mechanical agitation, high-pressure homogenization, and sonication or bottom-up methods, based on direct drop-by-drop generation, such as membrane emulsification ${ }^{22-24}$, microchannel emulsification, ${ }^{25}$ and microfluidic techniques. ${ }^{26-29}$ Conventional emulsification methods are energy intensive and often lead to polydispersed microspheres. Microfluidic methods allow production of particles with low polydispersity $(\mathrm{CV}=$ Standard Deviation/Mean $<3 \%)$ and high encapsulation efficiency that are perfectly tailored to meet the needs of pharmaceutical industry. ${ }^{30}$ However, microfluidic droplet generators are typically planar (2-D) and made 
from single crystal silicon chips using expensive microfabrication methods ${ }^{31}$ or mouldable polymers such as polydimethylsiloxane (PDMS), which swell and deform in contact with organic solvents. ${ }^{29}$

Here, we investigate formation of structured polymeric microspheres using glass capillary microfluidic devices. These devices pioneered by Utada et al. ${ }^{32}$ offer 3-D flow focusing that minimizes wetting, they are cheap to fabricate and more mechanically robust and inert than polymeric devices. ${ }^{33,13}$ Microstructural modifications of polymer matrix occurred due to introduction of nanoclay, non-solvent or second polymer, which resulted in the formation of composite and non-spherical particles and particles with structured surface (Figure 1b-d).

(a)
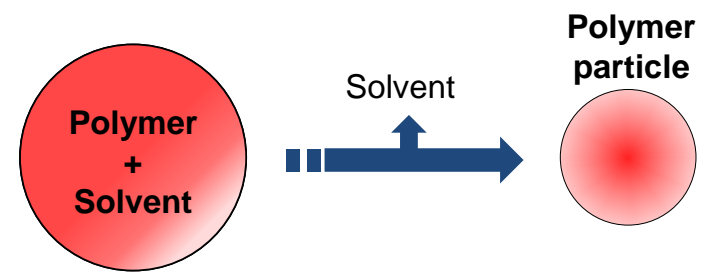

(b)
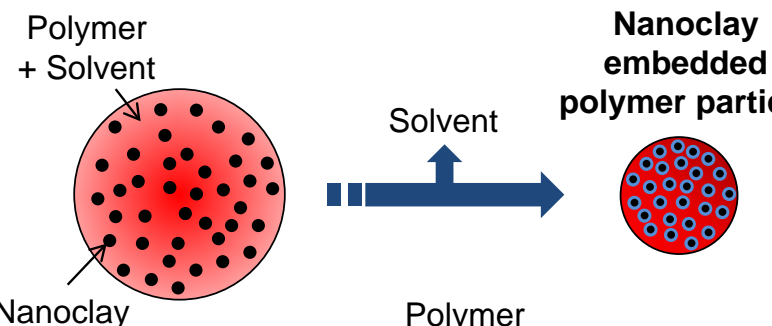

embedded

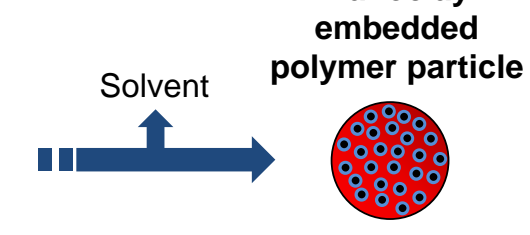

$$
\text { Nanoclay }
$$

Polymer

+ Solvent

Golf ball-

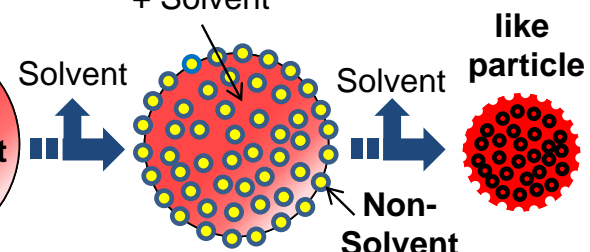

(c)

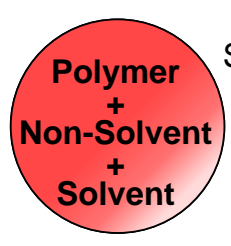

(d)

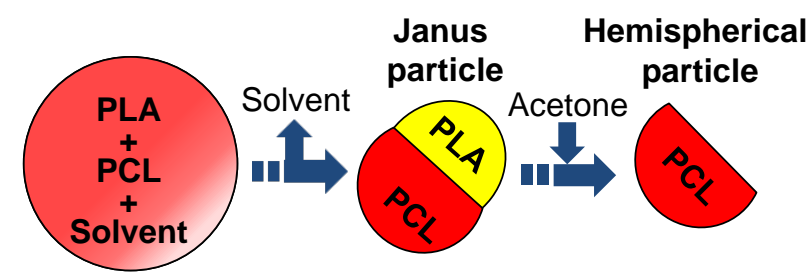

Figure 1. Strategies used in this work for creation of polymer particles: (a) Plain polymer particle; (b) Nanoclay embedded polymer particle; (c) Golf ball-like particle; (d) Janus and hemispherical particle. 
A special attention was paid on the effect of flow conditions on the retention (trapping) of suspended nanoparticles in the device. This phenomenon is important in all microfluidic processes where fluid streams entering a microfluidic channel contain suspended nanoobjects, such as colloidal particles, nano-assemblies, nanofibers, nanotubes or viruses. The effect of internal structure of the particles on the release rate of encapsulated drug was also discussed. Glass capillary devices have been used for fabrication of vesicles such as colloidosomes ${ }^{34}$, polymersomes, ${ }^{35}$ and liposomes, ${ }^{36}$ and microparticles such as solid $\operatorname{lipid}^{37}$ and microgel ${ }^{33}$ particles.

This paper presents new applications of glass capillary microfluidics for the production of drug delivery vehicles composed of nanoclay-embedded, golf ball-like, Janus, and hemispherical polymeric particles. A facile control over the particle size and morphology achieved in this work enables accurate prediction of drug release behavior.

\section{EXPERIMENTAL SECTION}

Materials. Poly(dl-lactic acid) (PLA, Ingeo ${ }^{\mathrm{TM}}$ 4060D, $\mathrm{M}_{\mathrm{w}}=89,000 \mathrm{gmol}^{-1}$ ) supplied by NatureWorks LLC (Minnetonka, MN, USA), poly(lactic-co-glycolic acid) (PLGA, $\mathrm{M}_{\mathrm{w}}=$ 10,000 $\mathrm{g} \mathrm{mol}^{-1}$, 50\% DL-lactic acid and 50\% glycolic acid), supplied by Wako Pure Chemical Industries (Osaka, Japan) and polycaprolactone $\left(\mathrm{PCL}, \mathrm{M}_{\mathrm{w}}=14,000 \mathrm{~g} \mathrm{~mol}^{-1}\right)$ supplied by Sigma-Aldrich (UK) served as primary ingredients of the microspheres. Organically modified nanoclay (Cloisite ${ }^{\circledR}$ 30B, $\rho=1980 \mathrm{~kg} \mathrm{~m}^{-3}$, Southern Clay Products, Gonzales, TX, USA) consisting of plate-like montmorillonite nanoparticles surface-substituted with alkyl 
ammonium ions, was used as a polymer filler for the microspheres. Dichloromethane (DCM, HPLC grade, Fisher Scientific, UK) and ethyl acetate (Sigma-Aldrich, UK) were used as nonpolar polymer solvents. 2-Methylpentane (Sigma-Aldrich, UK) was added to the dispersed phase as a polymer non-solvent in the production of golf ball-like particles. Paracetamol (4acetamidophenol) obtained from Sigma-Aldrich was used for drug release experiments. Poly(vinyl alcohol) (PVA, $\mathrm{M}_{\mathrm{w}}=13,000-23,000,87-89 \%$ hydrolyzed) supplied by SigmaAldrich (UK) was used as a water-soluble stabilizer in the continuous phase. Reverse osmosis water was prepared using Millipore 185 Milli-Q Plus apparatus. Rhodamine 6G (Rh6G) and Nile red (Sigma-Aldrich, UK) were used as fluorescence dyes for identification of bifacial particle morphology. 2-[methoxy(polyethylenoxy)propyl]-trimethoxysilane (MPEOPS) supplied by Fluorochem Ltd (UK) was used to enhance hydrophilicity of inner capillary.

Methods. Fabrication of Glass Capillary Device. A round capillary with inner diameter 0.58 $\mathrm{mm}$ and outer diameter $1 \mathrm{~mm}$ (Intracel, UK) was pulled using a micropipette puller (Sutter Instrument Company, USA). The tip of the pulled capillary was polished to desired orifice size using an abrasive paper (Black Ice Waterproof T402 Paper, Alpine Abrasives, UK), cleaned with compressed air and Milli-Q water and treated with MPEOPS. A 150-mm long square capillary with an inner diameter of $1.05 \pm 0.1 \mathrm{~mm}$ (Atlantic International Technologies, USA) was cut with a pen cutter to a required length of $50 \mathrm{~mm}$ and attached to a microscopic slide using epoxy glue (5-Minute Epoxy ${ }^{\circledR}$ Devcon). The round capillary was then inserted in the square capillary and centred using an inverted microscope (XDS-3, GX Microscopes, UK) before it was glued to position. The exposed end of the round capillary was connected via PVC medical tubing (ID: $1.00 \mathrm{~mm}$, OD: $1.05 \mathrm{~mm}$; Fisher Scientific, UK) to collection 
vial. All fabrication steps were carried out in a dust-free environment and disposable medical gloves were worn to prevent contamination of the device.

Production of emulsions and particles. The compositions of dispersed phase used in the production of different particles are shown in Table 1.

Table 1. Composition of dispersed phase used in this work for preparation of different particles. The continuous phase was $5 \mathrm{wt} \%$ PVA in Milli-Q water in all formulations.

\begin{tabular}{lll}
\hline Particle structure & Dispersed phase composition & wt\% \\
\hline \multirow{2}{*}{ Plain polymer particles } & Polymer (PLA or PLGA) & $0.5,1$ or 2 \\
& Solvent (DCM or ethyl acetate) & $99.5,99$ or 98 \\
\hline \multirow{2}{*}{ Nanoclay-embedded polymer } & Nanoclay & 0.02 or 0.04 \\
particles & Polymer (PLA or PLGA) & 0.98 or 0.96 \\
& Solvent (DCM or ethyl acetate) & 99 \\
\hline \multirow{2}{*}{ Golf ball-like polymer particles } & Nonsolvent (2-methylpentane) & 0.3 or 3 \\
& Polymer (PLA or PLGA) & 0.7 or 7 \\
& Solvent (DCM or ethyl acetate) & 99 or 90 \\
\hline \multirow{2}{*}{ Janus polymer particles } & Polymer mixture (PCL + PLA) & 3 \\
& (PCL:PLA = 1:2 or 2:1 v/v) & \\
& Solvent (DCM) & 97 \\
\hline
\end{tabular}

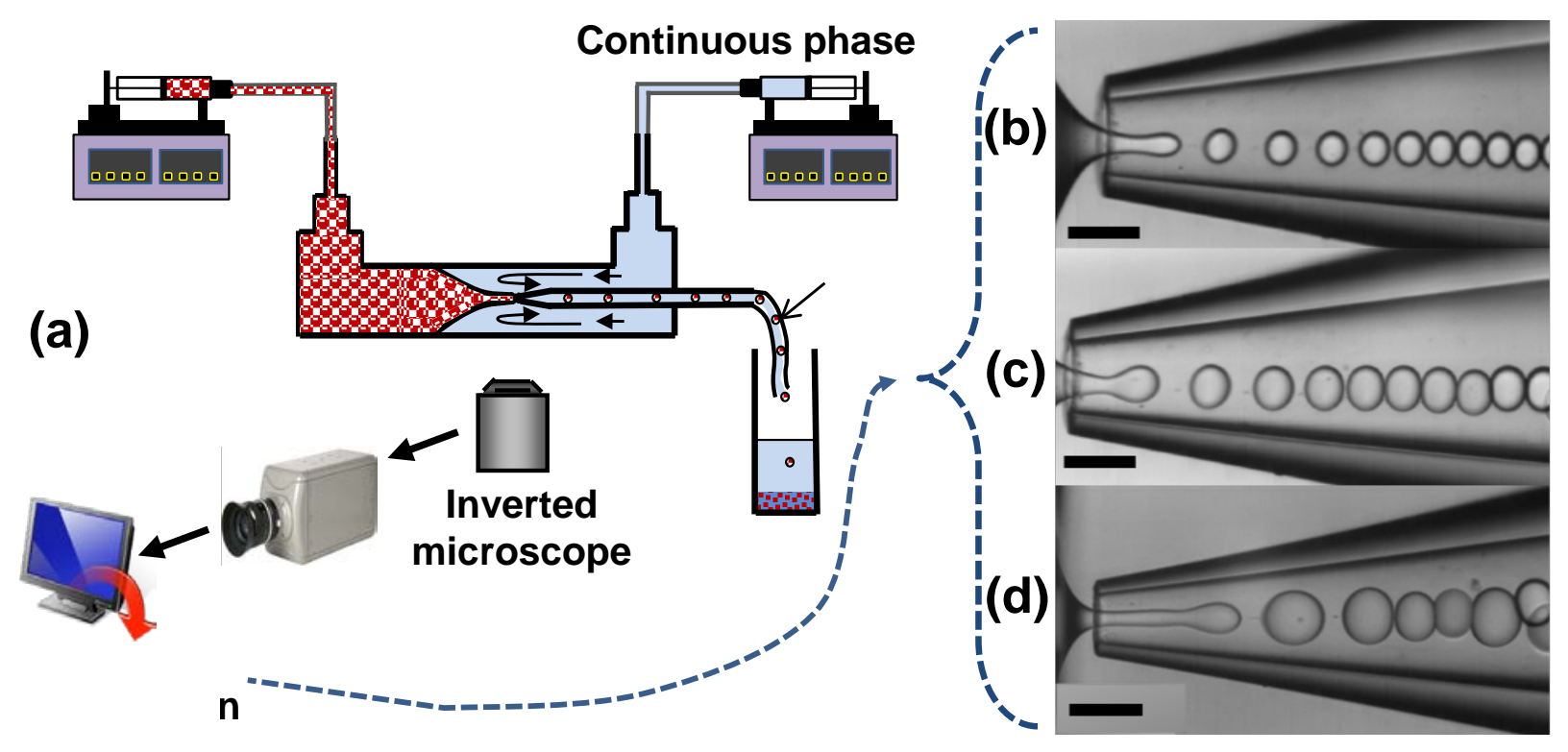


Figure 2. (a) Experimental setup of microfluidic device for the production and monitoring of microdroplet generation; (b) and (c) Monodispersed droplet generation in the dripping regime for a dispersed phase of PLA/nanoclay in DCM; (d) Polydispersed droplet generation in the jetting regime for dispersed phase of PLA in DCM. Scale bars: $250 \mu \mathrm{m}$.

For droplet production, glass capillary device was placed on a GXM XD63 optical inverted microscope. The dispersed and continuous phase of desired formulation (Table 1) were delivered from two gas tight syringes with Luer-lock fitting (VWR Catalyst Company, UK) mounted on separate Harvard Apparatus 11 Elite syringe pumps (Figure 2). PTFE medical tubing (Smiths Medical International Ltd, UK) and polyethylene medical tubing (Fisher Scientific, UK) were used to deliver the dispersed and continuous phase respectively to the corresponding needle on the device. The continuous phase was delivered via a 3-way stop cock (VWR Catalyst Company, UK) and Puradisc syringe filter (Sigma-Aldrich Company, UK) for easy syringe refilling and removal of microscopic impurities in the liquid. The two phases were supplied within the device counter-currently at low flow rates, such that the oil/aqueous interface slowly approached the orifice of the inner capillary to avoid wetting the tip by the oil phase. Droplet formation occurred on adjustment flowrates with syringe pumps.

Droplets were generated within a tapered section of the collection capillary (Figure 2 b-d). Real-time monitoring of droplet generation was achieved with a high speed camera (Phantom V9.0, Vision Research, USA) supported by the Phantom Camera Control (PCC) software (version 2.14.727.1, Vision Research, USA), which provided interface for image acquisition, brightness/contrast adjustment, and post-generation analysis. Generated droplets were collected in a vial and vacuum evaporated at room temperature to form particles. 
Analysis of Video Recordings. Recorded videos of droplet generation were stored in the camera's internal memory with the aid of the PCC software. The number of frames per second $n_{s}$ and the number of frames stored $n_{f}$ were chosen based on the storage space availability and droplet generation rate. The droplet generation frequency $f$ was calculated as: $f=n_{d} n_{s} / n_{f}$, where $n_{d}$ is the number of droplets generated in $n_{f} / n_{s}$ seconds, determined using ImageJ v.1.44 software (Wayne Rasband, National Institute of Health).

$X$-Ray Diffraction (XRD) Analysis. Wide angle X-ray diffraction patterns were obtained using a Bruker D2 Phaser diffractometer fitted with a 1-dimensional LynxEYETM detector. A copper X-ray source $(\mathrm{K} \alpha=1.54184 \AA)$ was used run at $30 \mathrm{kV}$ and $10 \mathrm{~mA}$, with $\mathrm{K} \beta$ radiation suppressed by means of a $0.5 \mathrm{~mm}$ thick nickel filter. Patterns were recorded over a $2 \theta$ range of $2-40^{\circ}$ with a step size of $0.02^{\circ}$ and an equivalent step time of $49.2 \mathrm{~s}$. Sample rotation was set at 15 rpm. Bruker's proprietary Eva 2.0 software was used to obtain the spectra.

Scanning Electron Microscopy (SEM) and Focused Ion Beam (FIB) Imaging. Imaging was carried out using a dual-beam focused ion beam scanning electron microscope (FIB-SEM) (Nova 600 Nanolab, FEI Company, Hillsboro, Oregon, USA). All SEM imaging were carried out at $10 \mathrm{kV}$ and $2.1 \mathrm{nA}$ over a $30 \mu$ aperture exposure.

For FIB imaging $30 \mathrm{kV}$ was used throughout. External angular imaging was initially obtained at $30 \mathrm{pA}$ before an increase to $0.3 \mathrm{nA}$ for a protective platinum (Pt) deposit. A cross-section was then obtained at $20 \mathrm{nA}$ before cross section cleaning was carried out at $7 \mathrm{nA}$ and reduced to $3 n A$ for a final cleaning. Cleaning was necessary for better microstructural imaging. The current was finally reduced to $30 \mathrm{pA}$ to preserve the exposed cross-sectional microstructure 
and prevent modification due to beam exposure during imaging, which was carried out at approximately 2 min/image for noise reduction.

Confocal Laser Scanning Microscopy (CLSM). The microstructure of Janus particles was vizualised using a Nikon Eclipse TE300 confocal inverted microscope connected to a computer running Zeiss LaserSharp 2000 ${ }^{\mathrm{TM}}$ software. The Janus particles were stained with Rhodamine 6G and Nile Red, added to the dispersed phase prior to emulsification. A suspension of Janus particles to be scanned was placed on a microscopic slide and allowed to dry. The sample was excited with argon laser with a wavelength of $488 \mathrm{~nm}$ and helium-neon laser with a wavelength of $543 \mathrm{~nm}$. The total emission was separated into two images captured by two photomultiplier tubes (PMTs): PMT1 captured fluorescence between $515 \pm 30$ nm (green region) and PMT2 captured fluorescence above 570 nm (yellow-red region).

Cumulative Drug Release Study. 0.7 wt\% of paracetamol (PCM) was added to the dispersed phase containing ethyl acetate (EA). The concentration of PCM in the continuous phase was measured using a Lambda 35 UV/Vis (PerkinElmer Instruments, UK) double beam spectrophotometer, supported by UVWINLAB version 2.85.04 software (PerkinElmer Instruments, UK), over $120 \mathrm{~h}$ at a wavelength of $242 \mathrm{~nm}$. The solubility of PCM in EA and water is 10.7 and $17.4 \mathrm{~g} / \mathrm{kg}$, respectively. ${ }^{38}$ The cumulative release was determined by:

$$
\text { Cumulative release }=\frac{C_{\text {Drug }}}{C_{\text {Drug }}^{0}} \times 100
$$

where $C_{D r u g}$ is the concentration of drug in the continuous phase and $C_{D r u g}^{0}$ is the predicted drug concentration in the continuous phase after complete release. 
Governing Equations and Numerical Modelling. Numerical modelling of drop generation was carried out using a two-dimensional axisymmetric volume of fluid (VOF) approach. ${ }^{39}$ The following equations of mass, Eq. 2, and momentum conservation, Eq. 3, were used:

$$
\begin{gathered}
\frac{\partial \rho}{\partial t}+\nabla \cdot(\rho \vec{U})=0 \\
\frac{\partial}{\partial t}(\rho \vec{U})+\nabla \cdot(\rho \vec{U} \vec{U})=-\nabla P+\nabla \cdot\left[\mu\left(\nabla \vec{U}+\nabla \vec{U}^{T}\right)\right]+\overrightarrow{F_{b}}
\end{gathered}
$$

where $\vec{U}$ and $P$ are velocity and pressure and $t, \mu$, and $\rho$ are time, dynamic viscosity and density. The interfacial force, $F_{\gamma}$, and gravitational force are included in the source term, $F_{b}$, However, since the length-scale is in the order of micro, the gravitational force is negligible and $\overrightarrow{F_{b}}=\overrightarrow{F_{\gamma}}$. In VOF model, a momentum equation is solved for all phases and the advection of interface is tracked by solving a transport equation for volume fraction, $f$ :

$$
\frac{\partial f}{\partial t}+\vec{U} \cdot \nabla f=0
$$

where f determines the portion of each cell filled with one of the two phases:

$$
\begin{array}{ll}
f=0 & \text { the cell is filled with aqueous phase } \\
0<f<1 & \text { fluid-fluid interface exists in the cell } \\
f=1 & \text { the cell is filled with oil phase }
\end{array}
$$

The dynamic viscosity and density in the momentum equation, Eq. 3, were calculated by:

$$
\begin{aligned}
& \mu=f \mu_{o}+(1-f) \mu_{w} \\
& \rho=f \rho_{o}+(1-f) \rho_{w}
\end{aligned}
$$

where the subscripts o and w refer to oil and aqueous phases respectively. Continuum surface force (CSF) method ${ }^{40}$ was used to calculate the interfacial force term included in Eq. 3:

$$
F_{\gamma}=\gamma \kappa \nabla f
$$


where $\gamma$ is the interfacial tension and $\kappa$ the local curvature of the interface, calculated as:

$$
\kappa=\nabla \cdot \hat{n}
$$

where $\hat{n}$ is the unit normal defined as:

$$
\hat{n}=\frac{\nabla f}{|\nabla f|}
$$

Equation 3 was solved using a pressure-based segregated algorithm in a finite-volume based commercial software Ansys ${ }^{\circledR}$ Fluent v. 15.0. Figure s.1 shows a schematic of the simulated geometry and the mesh used in the simulations. The discretised momentum equation was approximated by second order upwind scheme and the pressure term was interpolated using PRESTO scheme. The exact pressure was calculated on the cell faces in order to prevent interpolation error. The pressure-velocity coupling was achieved by SIMPLE scheme and the interpolation of interface was performed by Geo-Reconstruct algorithm. In order to reduce the computational cost, a variable time step method was used with $C o=0.35$. A grid independence study was carried out by generating four meshes with resolutions of 8, 6, 4, and $2 \mu \mathrm{m}$. Since the solutions for 4 and $2 \mu \mathrm{m}$ were very similar, the resolution of $4 \mu \mathrm{m}$ was selected for the simulations. A summary of the boundary conditions is listed in Table 2 .

Table 2. Summary of the boundary conditions used in this work.

\begin{tabular}{ll}
\hline Boundary & Type \\
\hline Inlets & Mass flow inlet \\
Outlet & Pressure outlet \\
Symmetry Axis & Axis \\
Wall & No-slip wall \\
\hline
\end{tabular}

Rheological Characterization. The densities of dispersed and continuous phases were determined at $25^{\circ} \mathrm{C}$ using a $10 \mathrm{ml}$ pycnometer. The kinematic viscosity measurements were 
performed using a U-tube viscometer at $25^{\circ} \mathrm{C}$. The interfacial tension was measured using a DSA100S drop shape analyzer (KRÜSS GmbH, Hamburg, Germany). The results are listed in Table 3.

Table 3. Physical properties of dispersed and aqueous phase used for numerical validation. The interfacial tension was measured against $5 \mathrm{wt} \%$ aqueous solution of PVA.

\begin{tabular}{lccc}
\hline Phase & $\begin{array}{c}\text { Density at } \mathbf{2 9 8} \\
\mathbf{K}\left(\mathbf{k g ~ d m}^{-3}\right)\end{array}$ & $\begin{array}{c}\text { Viscosity at } \\
\mathbf{2 9 8} \mathbf{~ K ~ ( m P a s )}\end{array}$ & $\begin{array}{c}\text { Interfacial } \\
\text { tension }\left(\mathbf{m N m}^{-\mathbf{1}}\right)\end{array}$ \\
\hline 1 wt\% PLGA in Ethyl Acetate & 0.910 & 0.5 & 1.0 \\
1 wt\% PLA/nanoclay (96:4) in DCM & 1.283 & 1.3 & 2.2 \\
5 wt\% PVA in water & 1.020 & 4.0 & - \\
\hline
\end{tabular}

\section{RESULTS AND DISCUSSION}

Production of Emulsions. Movie 1 in the supporting information shows a typical droplet generation behavior in dripping regime. Monodispersed droplets with a diameter of $130 \mu \mathrm{m}$ were generated using orifice with $200 \mu \mathrm{m}$ diameter. Droplets were stable against coalescence in the collection capillary. The droplet pinch off occurred at approximately one orifice diameter downstream of the orifice at the frequency of $410 \mathrm{~Hz}$. The droplet size and generation frequency were controlled by manipulating the flowrates and varying the orifice diameter of the collection tube.

Effect of Phase Flowrate Ratio. Figure 3 shows the effect of flow rate ratio, $Q_{c} / Q_{d}$ on the average droplet diameter, $D_{d}$ for three different orifice diameters, $D_{o}$. The effect of $Q_{c} / Q_{d}$ on the coefficient of variation of droplet sizes, $C V$ and the droplet generation frequency, $f$ is shown on the same figure for $D_{o}=200 \mu \mathrm{m}$. The dispersed phase was $1 \mathrm{wt} \% \mathrm{PLA} /$ nanoclay mixture in DCM with a polymer to nanoclay ratio of 98/2 (w/w). 

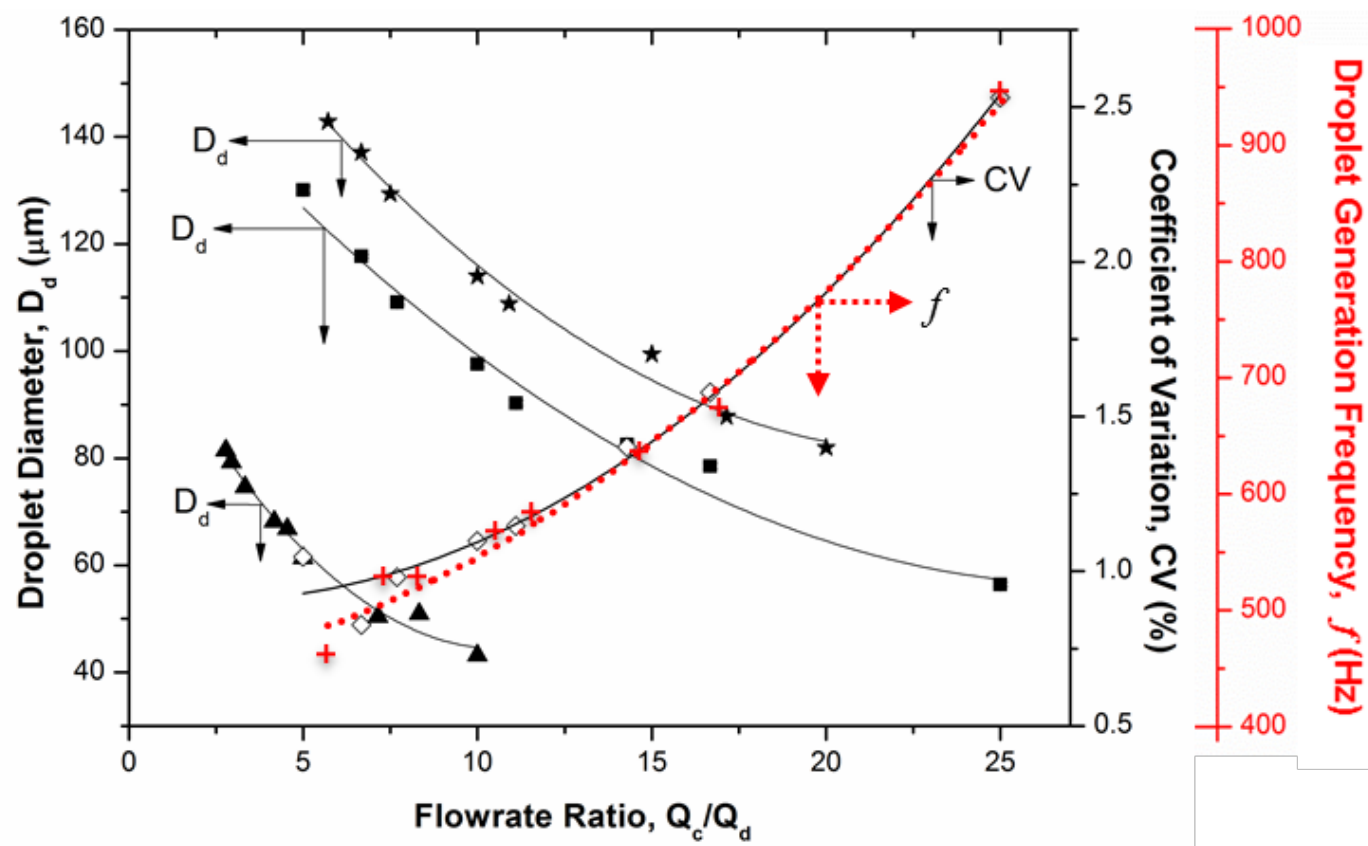

Figure 3. The effect of continuous to dispersed phase flow rate ratio, $Q_{d} / Q_{d}$ on average droplet diameter, $D_{d}$ in dripping regime: $\star D_{o}=224 \mu \mathrm{m}, Q_{c}=12 \mathrm{ml} \mathrm{h}^{-1} ; \mathbf{\square} D_{o}=200 \mu \mathrm{m}, Q_{c}$ $=10 \mathrm{ml} \mathrm{h}^{-1} ; \boldsymbol{\Delta} D_{o}=100 \mu \mathrm{m}, Q_{c}=5 \mathrm{ml} \mathrm{h}^{-1}$. The coefficients of variation of droplet sizes, $C V$ $(\diamond)$ and droplet generation frequencies, $f(+)$ are shown for $200 \mu \mathrm{m}$ orifice size only. The dotted line is for $f$. The dispersed phase is $1 \mathrm{wt} \%$ PLA/nanoclay (98/2) in DCM.

The droplet size was found to decrease with an increase in the flow rate ratio. When the data were plotted as $D_{d} / D_{o}$ vs. $Q_{c} / Q_{d}$ on a log-log graph, a single master line was obtained for all orifice sizes with the following equation: $D_{d} / D_{o}=A\left(Q_{c} / Q_{d}\right)^{B}$, where $A=1.23, B=-0.41$, and $R^{2}=0.940$ (the graph not shown here). The $B$ value of -0.41 is very similar to -0.39 found earlier for the dispersed phase composed of PLA in DCM, ${ }^{41}$ which means that the presence of nanoclay in DCM did not affect droplet generation. Droplet formation is a result of the balance between the detaching forces acting in the direction of flow (viscous drag and inertial force) and the interfacial tension force acting in the opposite direction. In dripping regime, which occurs at low flow rates of both phases, the main competition is between drag and interfacial tension force. A viscous drag force increases with increasing $Q_{c} / Q_{d}$, as a 
result of increase in the velocity gradient at the interface, causing the growing droplet to detach more quickly, which leads to smaller droplets and faster droplet generation (Figure 3). The ratio between viscous drag force exerted on the interface by the continuous phase and interfacial tension force is represented by the Capillary number of the continuous phase, $C a_{c}$, given by $\mu_{c} u_{c} / \sigma_{c}$, which is ranges between 0.003 and 0.006 for the conditions shown in Figure 3.

The results in Figure 3 were obtained in dripping regime, which occurs at $3<Q_{c} / Q_{d}<30$, but $Q_{d}$ must be within a certain range. If $Q_{d}$ is too low, droplet generation is unstable or nonexisting, irrespective of $Q_{c} / Q_{d}$ value. If $Q_{d}$ is too high, transition from dripping to widening jetting occurs and the droplet size is a result of the competition between inertial force of the dispersed phase and interfacial tension. The Weber number of the dispersed phase is the ratio between the inertial force of the dispersed phase and interfacial tension, $\sigma: W_{d}=\rho_{d} D_{j} u_{d}^{2} / \sigma$, where $\rho_{d}$ is the dispersed phase density, $D_{j}$ is the jet diameter, and $u_{d}$ is the mean velocity of the dispersed phase. For the data shown in Figure 3, $W e_{d}=0.002-0.02$.

At $Q_{c} / Q_{d}>30$, the dispersed phase forms a long narrow jet that breaks into polydispersed droplets further downstream (Figure 2d), which is known as the narrowing jetting regime. ${ }^{33}$ In contrast, dripping regime is characterised by highly uniform droplets with $C V<3 \%$ (Figs. 2 b and c). In Figure 3, $C V$ ranged from 0.8 to $2.5 \%$ for $D_{o}=200 \mu \mathrm{m}$ and similar $C V$ s were obtained in dripping regime for other orifice sizes and emulsion formulations.

As shown in Figure 3 for the dispersed phase containing DCM, progressively smaller droplets were generated when the orifice diameter was reduced from 224 to $100 \mu \mathrm{m}$. This effect is illustrated for ethyl acetate on the images shown in Figure 4, where the orifice diameters of 
$200,110,70$, and $40 \mu \mathrm{m}$ resulted in the droplet diameters of $149,69,47$, and $27 \mu \mathrm{m}$ respectively. In each case, droplets were smaller than their orifices preventing surface wetting. To generate droplets in dripping regime, $Q_{c} / Q_{d}$ was 3.6-12.5 and $Q_{d}$ was gradually reduced with reducing orifice size to prevent too high velocity of the dispersed phase at the orifice that would cause transition from dripping to widening jetting.

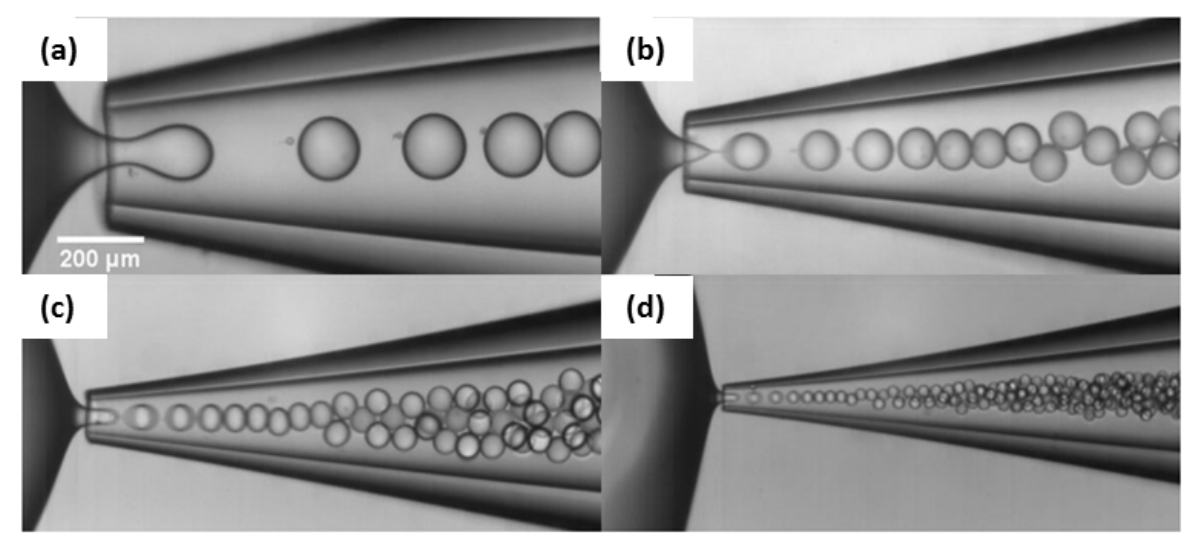

Figure 4. High speed camera stills of droplet generation showing variation of droplet size with orifice size: (a) $Q_{d}=1.5 \mathrm{ml} \mathrm{h}^{-1}, Q_{c}=8 \mathrm{ml} \mathrm{h}^{-1}, D_{o}=200 \mu \mathrm{m}, D_{d}=144 \mu \mathrm{m}$; (b) $Q_{d}=0.25$ $\mathrm{ml} \mathrm{h}^{-1}, Q_{c}=0.9 \mathrm{ml} \mathrm{h}^{-1}, D_{o}=100 \mu \mathrm{m}, D_{d}=69 \mu \mathrm{m}$; (c) $Q_{d}=0.1 \mathrm{ml} \mathrm{h}^{-1}, Q_{c}=0.5 \mathrm{ml} \mathrm{h}^{-1}, D_{o}=70$ $\mu \mathrm{m}, D_{d}=47 \mu \mathrm{m}$; (d) $Q_{d}=0.02 \mathrm{ml} \mathrm{h}^{-1}, Q_{c}=0.25 \mathrm{ml} \mathrm{h}^{-1}, D_{o}=40 \mu \mathrm{m}, D_{d}=27 \mu \mathrm{m}$. The scale bar displayed is the same for all figures. The dispersed phase is $1 \mathrm{wt} \% \mathrm{PLA} /$ nanoclay in ethyl acetate.

Nanoparticle filtration. A decrease in PLA to nanoclay ratio from 98/2 to 96/4 resulted in aggregation of nanoclay particles and a particle trapping in the dispersed phase in front of the orifice at low dispersed phase flow rates. An increase in the dispersed phase flow rate from 0.5 to $3 \mathrm{ml} \mathrm{h}^{-1}$ suppressed the particle trapping, as shown in Figs. 5(a.4-5). A VOF-CSF numerical model was developed to further study the drop generation behavior and get an indepth understanding of the underlying physics behind the trapping of particles at variable $Q_{d}$ values. The numerical model was validated against experimental data for the generation of W/O/W emulsions in glass capillary devices. ${ }^{42,43}$ The validation of the numerical model with 
current experiments with $\mathrm{O} / \mathrm{W}$ emulsions is provided in Figure 5 (a.1\&2 and b.1\&2). It can be seen that the numerical model is capable of reproducing the experimental behavior accurately.

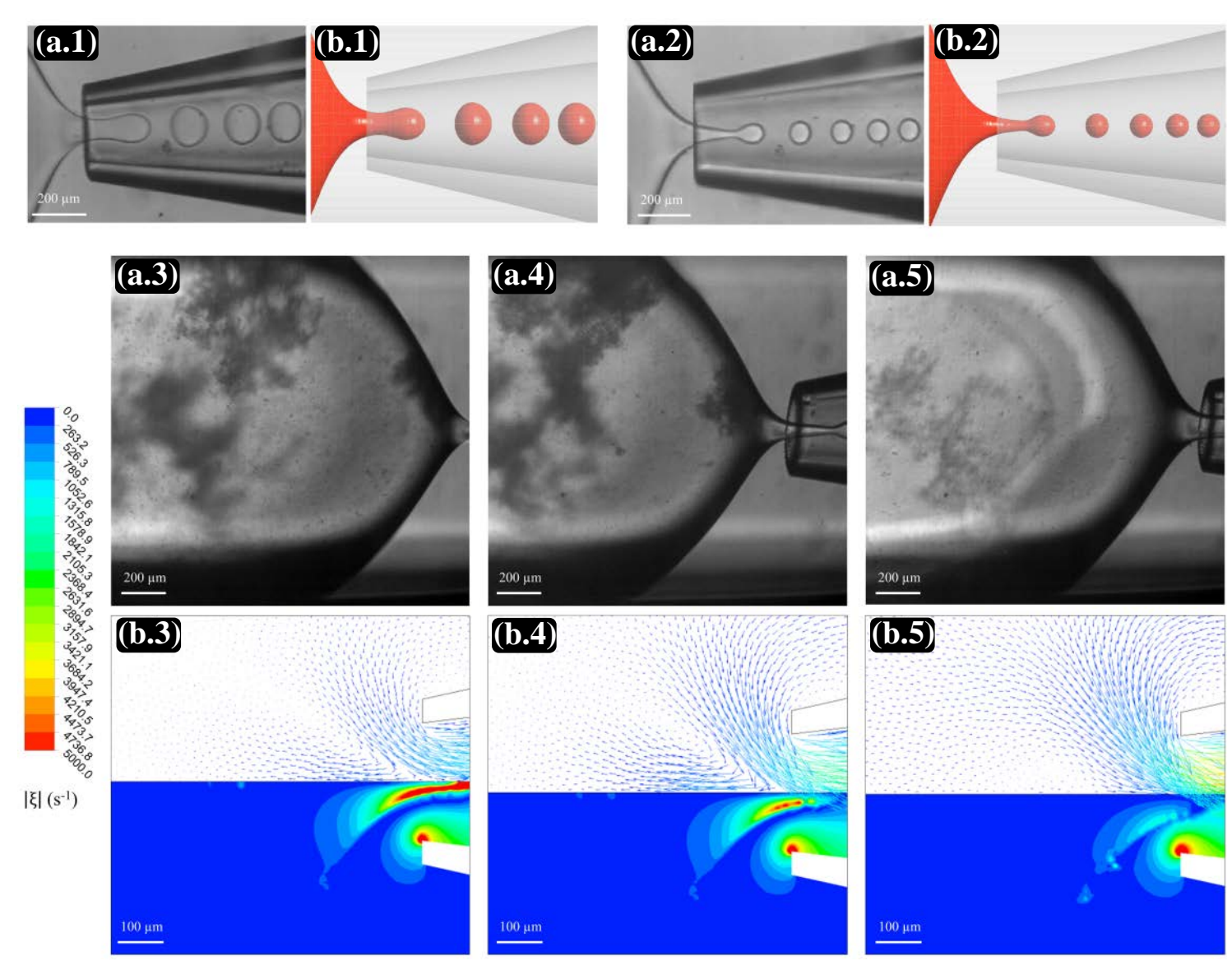

Figure 5. A qualitative comparison of the experimental and numerical drop generation behaviour. (a.1\&2): 1\%PLGA in ethyl acetate (a.1) $Q_{c}=6 \mathrm{ml} / \mathrm{h}, Q_{d}=1.5 \mathrm{ml} / \mathrm{h}$; (a.2) $Q_{c}=5$ $\mathrm{ml} / \mathrm{h}, Q_{d}=0.5 \mathrm{ml} / \mathrm{h}$; (а.3-5): 1\% PLA/nanoclay (w:w=96:4); (а.3) $Q_{c}=10 \mathrm{ml} / \mathrm{h}, Q_{d}=0.2 \mathrm{ml} / \mathrm{h}$; (a.4) $Q_{c}=10 \mathrm{ml} / \mathrm{h}, Q_{d}=0.5 \mathrm{ml} / \mathrm{h}$; (a.5) $Q_{c}=10 \mathrm{ml} / \mathrm{h}, Q_{d}=3 \mathrm{ml} / \mathrm{h}$. The numerical images in Figures (b.3), (b.4) and (b.5) include flow vectors (upper half) and vorticity magnitude contours (lower half).

Figures 5 (b.3-5) show the vorticity magnitude contours and flow vectors at the entrance of the collection tube at different $Q_{d}$ values, corresponding to Figs. 5 (a3-5). In each frame, the upper part shows flow vectors and the lower part shows vorticity magnitude contours. Vorticity is the measure of a fluid element rotation as it moves in flow field, and is defined as 
the curl of velocity vector: $\xi=\nabla \times \vec{U}$. Therefore, the vorticity magnitude $|\xi|$, is the magnitude of velocity vector curl, $|\nabla \times \overrightarrow{\mathrm{U}}|{ }^{44}$ At low dispersed phase flow rate of $Q_{d}=0.2 \mathrm{ml} \mathrm{h}^{-1}$ (Figure 5.a3 and b3), a very strong vortex flow forms in front of the orifice, which prevents the entrance of nanoparticles through the orifice. By increasing $Q_{d}$ from 0.2 to 0.5 to $3 \mathrm{ml} \mathrm{h}^{-1}$, the vortex flow strength decreases, which results in a reduction of vorticity magnitude and suppression of the filtration effect, so that progressively higher proportion of nanoparticles can pass through the orifice. The presence of strong vortex flow at low $Q_{d}$ is a consequence of the high velocity gradient at the liquid-liquid interface. As $Q_{d}$ increases, the velocity gradient is reduced causing the vortices to weaken. The particles closer to interface can enter the orifice easier than the particles further away from the interface. It can be explained by a higher level of shearing in the interfacial region than in the bulk of the dispersed phase flow. Movies (2-4) in supporting information show this phenomenon.

A particle trapping phenomenon shown in Figure 5 can be used to achieve a continuous passive separation of nanoparticles from fluid streams. It differs from inertial particle separation in a Vortex Chip consisted of multiple expansion-contraction reservoirs, ${ }^{45,46}$ as hydrodynamic flow focusing does not require a prior stage of particle alignment by inertial focusing. A passive separation of nanoparticles by microfluidic flow focusing has the advantage over traditional filtration of exploiting orifice which is significantly larger than the particles and therefore, not prone to clogging. It eliminates the need for frequent backwashing and maintenance shutdowns which are common in filtration processes.

Particle production from emulsions. Figure 6 shows images of different particles (nanoclay/PLGA, nanoclay/PLA, PLGA and PLA) produced by evaporation of ethyl acetate 
or DCM from droplets. The morphology and size of the particles depend on the dispersed phase composition and the initial droplet size. However, in each case, produced particles exhibited high size uniformity.
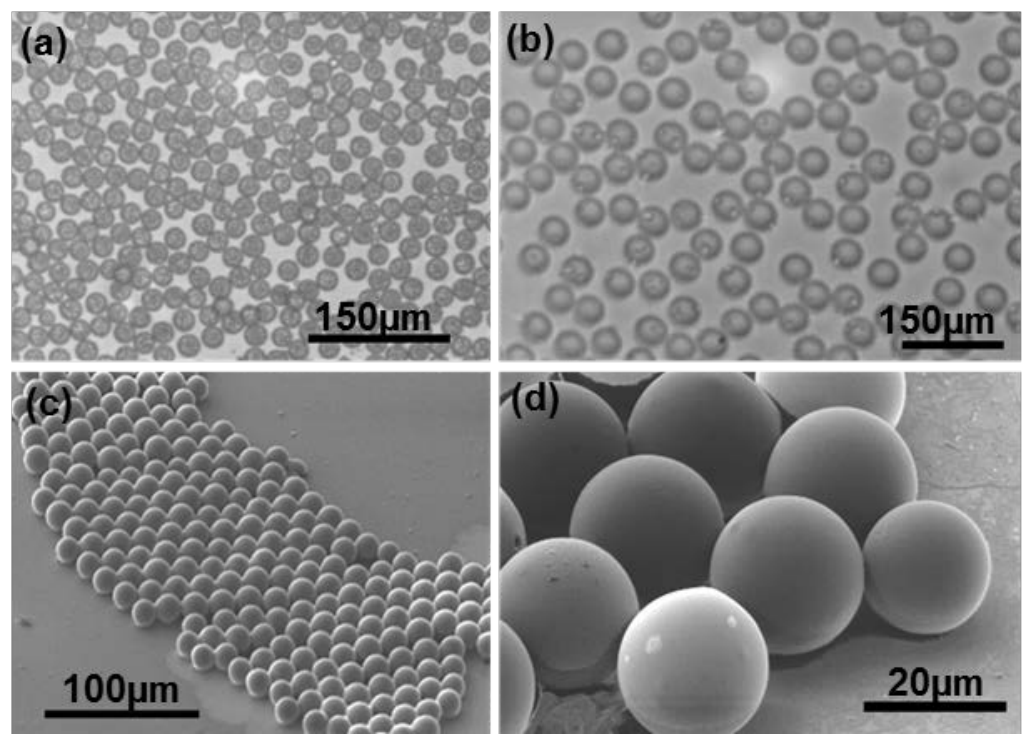

Figure 6. Monodispersed microparticles produced by evaporation of DCM or ethyl acetate from the generated droplets: (a) nanoclay embedded PLGA particles with $D_{p}=20 \mu \mathrm{m}$; (b) nanoclay embedded PLA particles with $\mathrm{D}_{\mathrm{p}}=40 \mu \mathrm{m}$; (c) plain PLGA particles with $\mathrm{D}_{\mathrm{p}}=16$ $\mu \mathrm{m}$; (d) plain PLA particles with $\mathrm{D}_{\mathrm{p}}=22 \mu \mathrm{m}$.

Effect of Dispersed Phase Composition. The size of the particles produced after complete solvent evaporation can be predicted from a material balance equation for non-volatile solids (polymer and nanoclay) in the dispersed phase. If the particles are nonporous, their volume $V_{p}$ and diameter $D_{p}$ are proportional to the initial volume $V_{d}$ and diameter $D_{d}$ of the droplets: ${ }^{41}$

$$
\begin{gathered}
V_{p}=\frac{x_{p} \rho_{d}}{\rho_{p}} V_{d} \\
D_{p}=\sqrt[3]{\frac{x_{p} \rho_{d}}{\rho_{p}}} D_{d}
\end{gathered}
$$


where $x_{p}$ is the mass fraction of non-volatile solids (polymer and nanoclay) in the dispersed phase, and $\rho_{p}$ and $\rho_{d}$ are the densities of the particles and droplets, respectively. Figure 7 shows the relationship between $D_{p}$ and $D_{d}$ for plain PLGA and PLA particles. The solid lines are the lines of best-fit to the experimental data, while the dotted lines correspond to Eq. (12), with their gradients equal to $\sqrt[3]{x_{p} \rho_{d} / \rho_{p}}$.
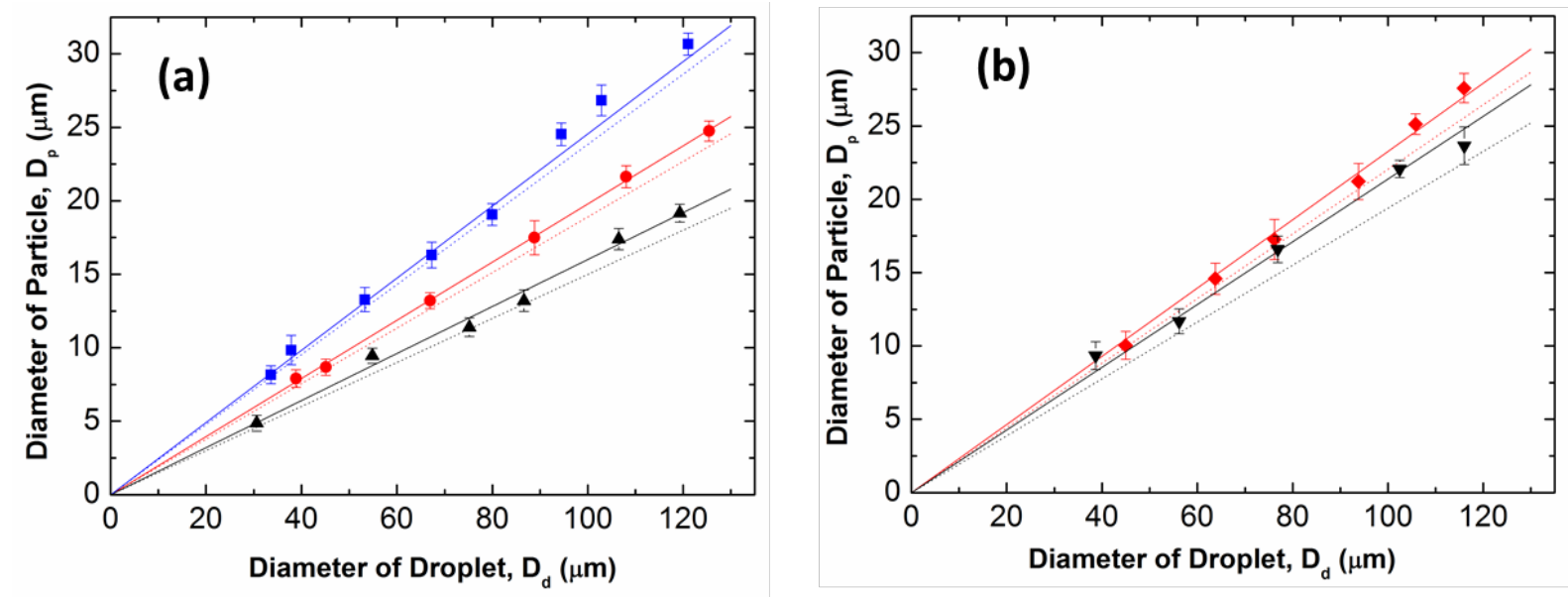

Figure 7. The particle diameter vs. droplet diameter for plain polymeric particles: (a) PLGA; (b) PLA. The dispersed phase formulation: $\Delta$ 0.5\% PLGA in EA, 1\% PLGA in EA, $\mathbf{2} \%$ PLGA in EA, 1\% PLA in DCM, and $\boldsymbol{\nabla} 1 \%$ PLA in EA. The solid lines are the best fit to the experimental data, while the dotted lines satisfy Eq. (12).

As predicted by Eq. (12), $D_{p}$ is directly proportional to $D_{d}$ for all formulations, with small deviations between the experimental and theoretical results for both polymers and solvents (DCM and EA). Figure 7 reflects the ability of the process to precisely tune the particle size over a wide range of 4-30 $\mu \mathrm{m}$, whilst maintaining high particle size uniformity. Particles that are $4 \mu \mathrm{m}$ are considered suitable for intravenous drug administration since the smallest capillary blood vessels have a diameter of about $7 \mu \mathrm{m} .{ }^{47}$ Particles in the size range of 10-30 $\mu \mathrm{m}$ are suitable for parenteral drug delivery via subcutaneous or intramuscular injection. ${ }^{48}$ 
As expected, the particle size increased with increasing the polymer concentration $x_{p}$, which can be seen in Figure 7(a) for PLGA particles at $x_{p}=0.5-2 \mathrm{wt} \%$. Under the same conditions, PLGA particles are smaller than PLA particles (Figures 7 (a) and (b) for $1 \mathrm{wt} \%$ polymer in EA), since particle diameters are inversely proportional to the cubed root of particle densities and the density of PLGA $\left(1.34 \mathrm{~g} \mathrm{~cm}^{-3}\right)$ is higher than that of PLA $\left(1.24 \mathrm{~g} \mathrm{~cm}^{-3}\right)$. The effect of solvent type on the particle size can be seen in Figure 7(b), which compares the size of PLA particles produced using the same polymer concentration in the dispersed phase (1 wt\%), but different solvents (EA and DCM). Smaller particles were produced using EA, due to the lower density of EA $\left(0.902 \mathrm{~g} \mathrm{~cm}^{-3}\right)$ as compared to DCM $\left(1.33 \mathrm{~g} \mathrm{~cm}^{-3}\right)$.

In Figure 7, a minor deviation between experimental particle sizes and their theoretical estimates is a result of pore formation in the polymer matrix, which was not accounted for in the material balance equation (Eq. 11). The particle porosity can be estimated from the ratio of the gradients of the theoretical and experimental $D_{p}$ vs. $D_{d}$ lines: ${ }^{13}$

$$
\varepsilon=1-\left[\left[\frac{\left(\partial D_{p} / \partial D_{d}\right)_{\text {theor }}}{\left(\partial D_{p} / \partial D_{d}\right)_{\text {exp }}}\right]\right]^{3}
$$

Solvent choice can also affect the porosity of particles, as can be seen in Figure 7(b). The porosity of PLA particles produced using DCM and EA was 14.8 and $25.5 \%$ respectively, estimated from Eq. (13). At $20^{\circ} \mathrm{C}$, water is more soluble in EA (3.3 wt\%) than in DCM (0.24 wt\%). ${ }^{49}$ The higher the solubility of water in the organic phase, the higher the amount of water taken up by the droplets and the higher the porosity of the particles after solvent evaporation, which is similar to the effect of internal water in $\mathrm{W} / \mathrm{O} / \mathrm{W}$ emulsion. ${ }^{13}$ Therefore, apart from phase flow rates and polymer concentration, densities of the polymer and solvent 
and water solubility in the organic phase also play a significant role in the control of particle size and porosity.

Nanoclay-Embedded Polymer Particles. Figure 8 shows the effect of nanoclay on the particle size for the dispersed phase containing PLA in DCM (red symbols) and PLGA in EA (black symbols). The solid lines are the trend lines for nanoclay embedded particles, the dashed lines are the trend lines for plain polymeric particles, while the dotted lines satisfy Eq. (12). For PLA in DCM, the $D_{p}$ vs. $D_{d}$ lines have higher gradients, due to higher density of DCM compared to EA. In the absence of swelling effects and particle porosity, all lines for the same polymer and solvent should coincide, irrespective of the presence of nanoclay (due to small amount of nanonclay in the polymer matrix), which is not the case in Figure 8.

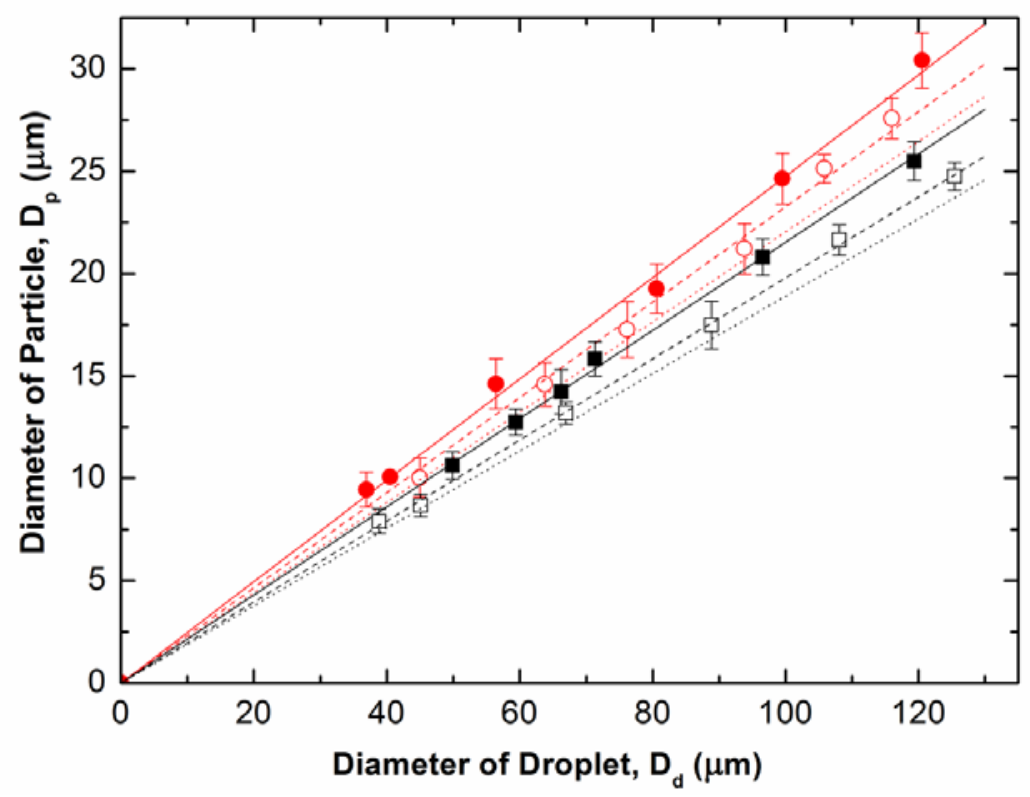

Figure 8. The particle diameter vs. droplet diameter for nanoclay embedded and plain polymeric particles: $\bigcirc$ Nanoclay embedded PLA, $\bigcirc$ Plain PLA, $\square$ Nanoclay embedded PLGA, $\square$ Plain PLGA. The PLA solvent was DCM and the PLGA solvent was EA. The polymer concentration was $1 \mathrm{wt} \%$ and the polymer/nanoclay mass ratio was 98:2. The solid lines are the trend lines for nanoclay embedded particles, the dashed lines are the trend lines for plain polymeric particles, while the dotted lines satisfy Eq. (12). 
A higher deviation of experimental particle diameters from their values predicted by Eq. (12) was observed for both nanoclay embedded polymers, as compared to plain particles. It can be explained by either higher porosity or higher degree of swelling of nanoclay-loaded particles compared to plain polymer particles.

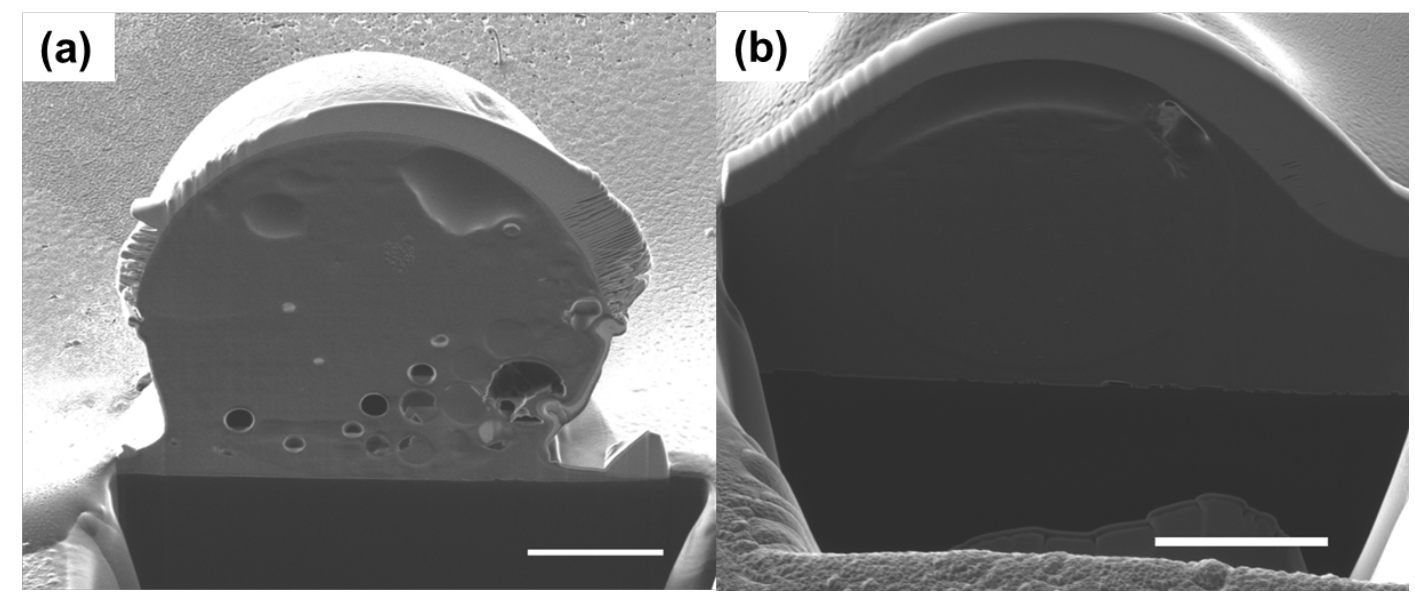

Figure 9. FIB cross-sections of: (a) PLA particle showing pores in the polymer network; (b) nanoclay embedded PLA particles with non-porous internal structure. Scale Bars: $5 \mu \mathrm{m}$.

FIB cross-sectioned images of PLA particles shown in Figure 9 indicate that nanoclayembedded PLA particles are significantly less porous than those produced without nanoclay. When placed in contact with water, montmorillonite can adsorb up to $10 \mathrm{~g} \mathrm{H}_{2} \mathrm{O} / \mathrm{g}$ clay, which can increase its volume by up to 20 times $^{50}$. The exact degree of swelling depends on the type of exchangeable cation. Although montmorillonites cation-exchanged with quaternary ammonium cations are primarily swollen by organic solvents, they can adsorb up to $400 \mathrm{mg}$ $\mathrm{H}_{2} \mathrm{O} / \mathrm{g}$ clay. ${ }^{51}$ This water uptake increases the size of the particles and makes the polymer matrix more compact and less permeable to gases. ${ }^{52}$ Hence, a compact structure of 
nanoclay/PLA particle in Figure $9 \mathrm{~b}$ is an evidence of the presence of nanoclay in the polymer matrix and the XRD plot shown in Figure 10 provides another evidence.

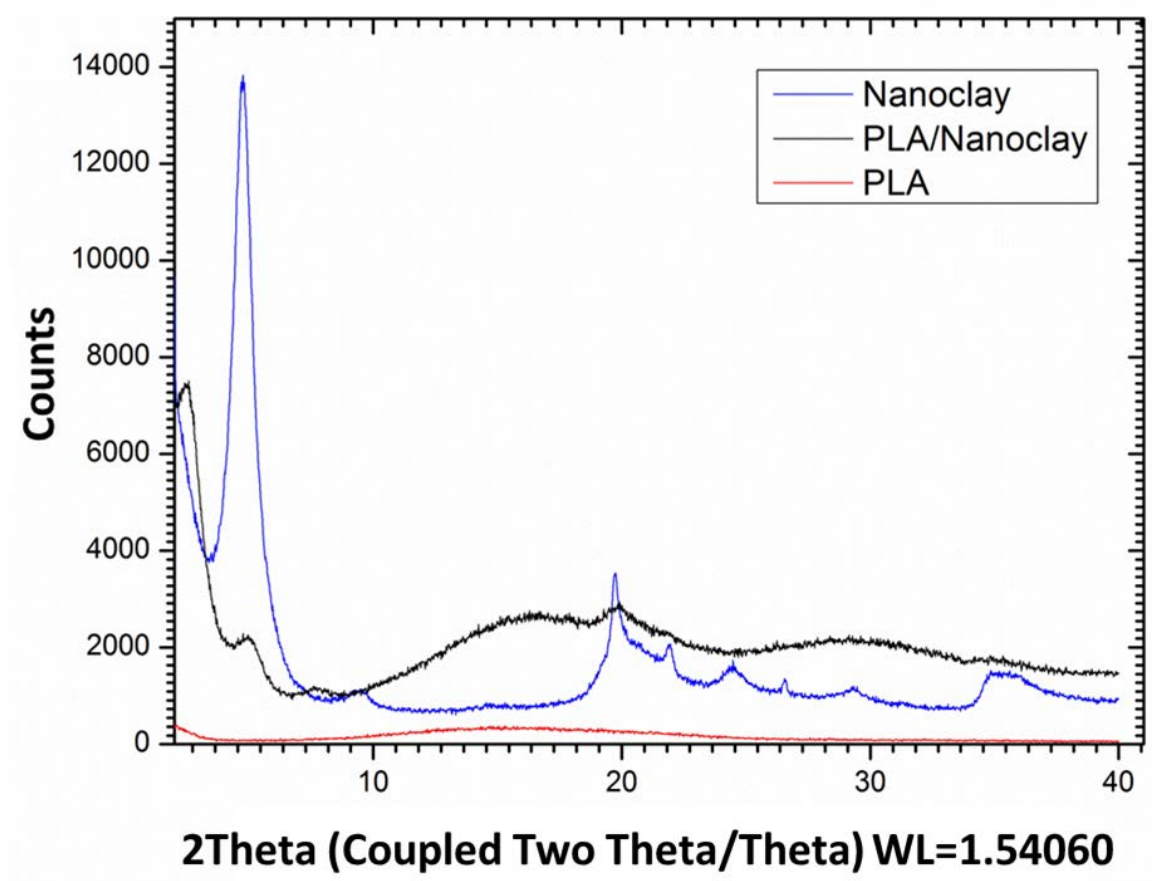

Figure 10. XRD plot of nanoclay powder, PLA/nanoclay particles and plain PLA particles.

The amorphous nature of pure poly(DL-lactic acid) is confirmed in Figure 10 by a broad amorphous 'hump' over a wide $2 \Theta$ range of $10-30^{\circ}$, due to X-rays being scattered in many directions. In contrast, high intensity narrower peaks were observed in the XRD pattern of pure crystalline nanoclay powder. Nanoclay embedded polymer particles exhibit crystal peaks at similar positions as pure nanoclay $\left(2 \Theta=5^{\circ}, 20^{\circ}, 30^{\circ}\right.$ and $\left.35^{\circ}\right)$, in addition to a wide hump part, which serves as an evidence of the embedment of nanoclay in the polymer matrix.

Golf-Ball Like Polymer Particles. The effects of introducing a polymer non-solvent (hydrocarbon porogen) in the dispersed phase are shown in Figure 11. Particles formed by phase separation within the droplets consisted of $1 \mathrm{wt} \%$ [2-methylpentane/PLA 30/70 (w/w)] 
in DCM are shown in Figure 11(a)-(c). During DCM evaporation, the droplets of 2methylpentane, since immiscible with PLA and less volatile than DCM, tend to heteroaggregate on the surface of PLA-laden evaporating droplets (see Figure 1c). ${ }^{11}$ This brings about dimples, which are 'footprints' formed by droplets of the non-solvent on the surface of the PLA particle. Also, pores can be seen in the FIB cross section shown in Figure 11 (d), which are the result of phase separation between the polymer and the non-solvent.

However, when the same emulsion was produced with gentle stirring in the collection vial, smaller dimples were formed on the surface of particles as shown in Figure 11(e) and (f). In this case, the droplets of the non-solvent detach earlier from the surface, thereby, inducing formation of smaller dimples on the surface. Pores are also noticeable in the particle cross section created by FIB (Figure 11g), since stirring does not affect the migration rate of inner droplets to the surface but only the rate of their detachment from the particle surface. 

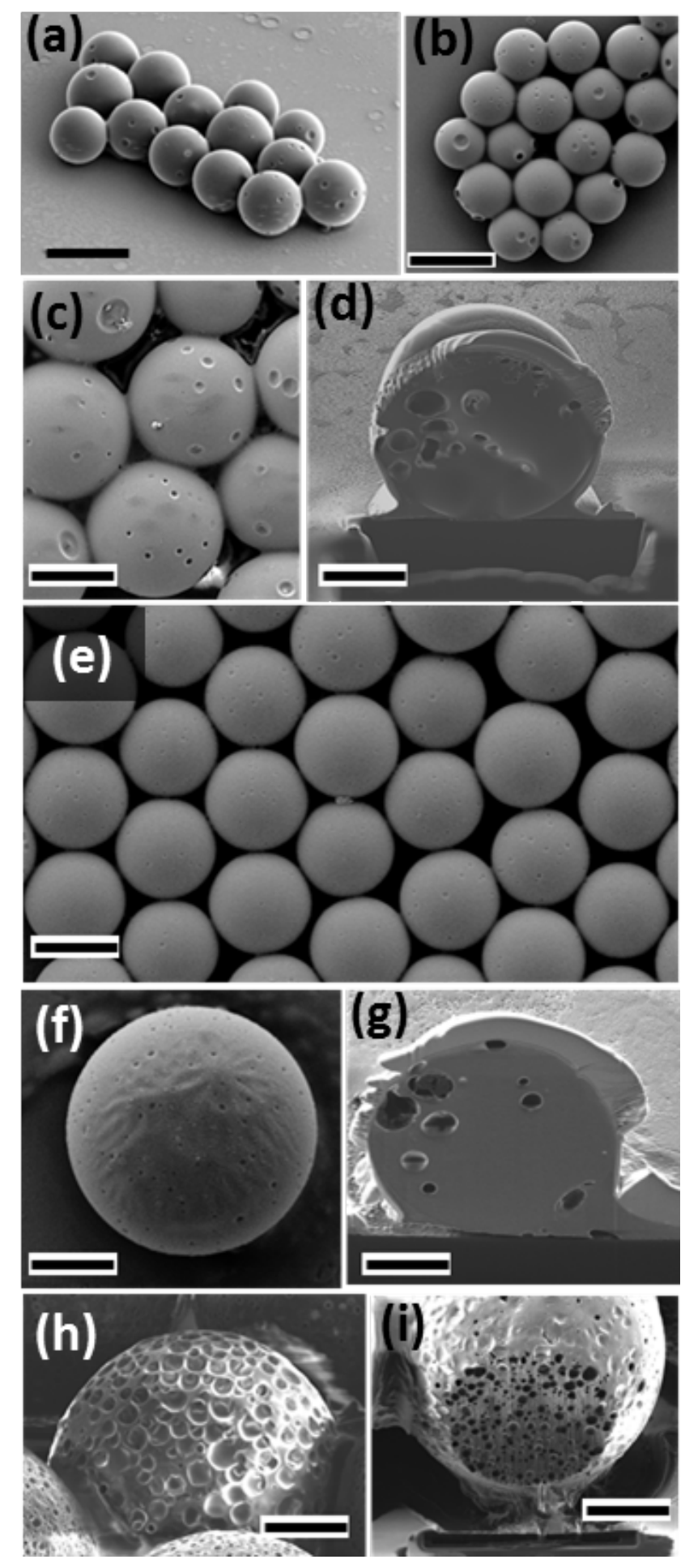

Figure 11. SEM images of dimpled PLA particles. The dispersed phase was: (a-g) 1 wt\% [2methylpentane/PLA (w:w=3:7)] in DCM without stirring (a-d) and with stirring (e-g) of the formed emulsion; (h-i) $10 \mathrm{wt} \% \%$ [2-methylpentane/PLA (w:w=3:7)] in DCM. Scale bars: (ab) $20 \mu \mathrm{m}$ (c) $9 \mu \mathrm{m}$ (d) $6 \mu \mathrm{m}$ (e) $20 \mu \mathrm{m}$ (f) $4.5 \mu \mathrm{m}$ (g) $44 \mu \mathrm{m}$ (h) $44 \mu \mathrm{m}$ (i) $50 \mu \mathrm{m}$. 
When $10 \mathrm{wt} \%$ [2-methylpentane/PLA(w:w=3:7)] in DCM was used as a dispersed phase with gentle stirring in the collection vial, microspheres with rougher surface were obtained, as shown in Figure 11(h) and (i). Higher particle porosity in Figure 11(i) can be attributed to the higher percent of non-solvent in the dispersed phase, as compared to that in Figure 11 (a-g). Therefore, the variation in the amount of polymer and non-solvent could serve as a means of adjusting particle porosity and surface roughness. Surface dimples can act as picoliter beakers, ${ }^{11}$ but can also enhance cell adhesion ${ }^{53}$ and particle mobility and dispersibility in fluids, due to Magnus effect and minimised contact area. It is important if the fabricated particles are intended to be used as carriers in dry powder inhaler formulations. ${ }^{54}$

Drug Release from Particles. The release patterns of paracetamol from the fabricated particles are shown in Figure 12. Increasing the diameter of plain PLGA particles from $17 \mu \mathrm{m}$ (Figure 12a) to $45 \mu \mathrm{m}$ (Figure 12b) reduced cumulative drug release after $1 \mathrm{~h}$ from 100 to 80 $\%$ with the remaining $20 \%$ released over $140 \mathrm{~h}$. It is generally accepted that higher surface area per unit volume leads to higher degradation rate of the polymer matrix. As shown in Figure 12(a), replacing PLGA with PLGA/nanoclay (98:2 w/w) in the dispersed phase further reduced paracetamol release, as a result of greatly increased drug diffusion path length due to the obstacles imposed by the impervious clay platelets. The relative permeability of polymer matrix depends on the volume fraction of the nanofiller in the host polymer, the aspect ratio of the platelets and their orientation. ${ }^{52}$ Improvement in drug release control is also observed in Figure 12b, which shows that the initial burst of drug release can be completely eliminated by incorporating nanoclay in the polymer matrix. 


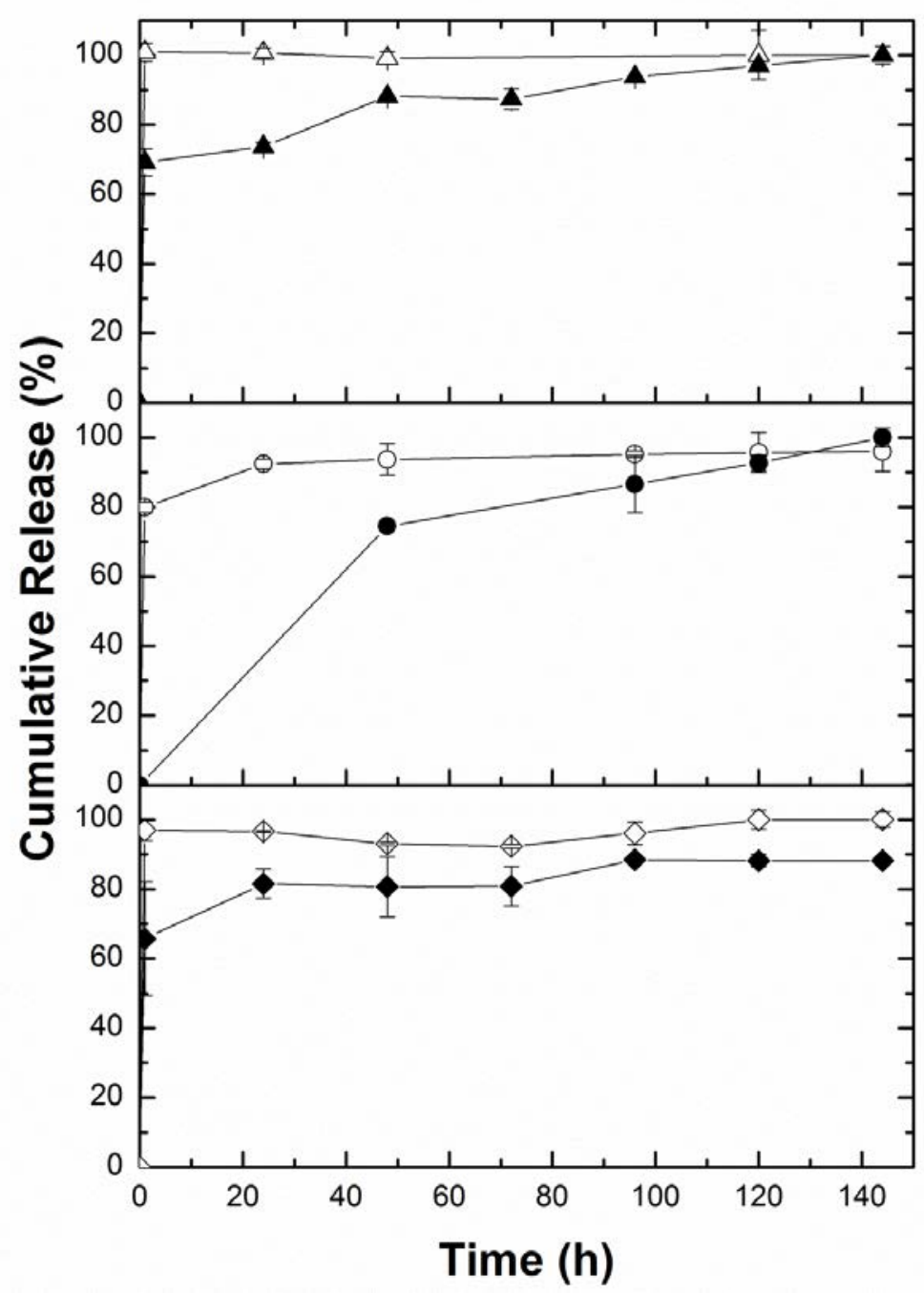

Figure 12. Release of paracetamol from microparticles: (a) $\Delta$ 17- $\mu \mathrm{m}$ plain PLGA, $\boldsymbol{\Delta} 17-\mu \mathrm{m}$ PLGA/nanoclay; (b) $\bigcirc$ 45- $\mu \mathrm{m}$ plain PLGA, $-45-\mu \mathrm{m}$ PLGA/nanoclay. The continuous phase: $5 \mathrm{wt} \%$ PVA in water saturated with ethyl acetate; (c) $\diamond 25-\mu \mathrm{m}$ golf ball-like PLA, $25-\mu m$ plain PLA. The continuous phase: 5 wt\% PVA in water. The dispersed phase used for production of plain PLGA and PLA particles: $2 \mathrm{wt} \%$ PLGA and $1 \mathrm{wt} \%$ PLA in ethyl acetate, respectively. The initial drug loading in dried particles: $5 \mathrm{wt} \%$. The error bars represent one standard deviation of the means based on 3 repeated measurements.

Furthermore, the amount of drug released after $1 \mathrm{~h}$ from plain 25- $\mu \mathrm{m}$ PLA particles (Figure 12c) was lower than that from plain $45-\mu \mathrm{m}$ PLGA particles (Figures 12b), although in the former case both the initial polymer concentration in the dispersed phase and the particle size was lower. It is expected because PLGA typically exhibits a faster degradation than PLA. ${ }^{49}$ 
PLA is more hydrophobic than PLGA due to extra methyl groups in glycolide residues and the ester bonds of PLA are less prone to hydrolysis due to steric hindrance. Furthermore, PLGA 50:50 used in this work has the fastest degradation rate among all PLGA copolymers. Figure 10c shows that the drug release rate from golf-ball-shaped PLA particles was higher than that from plain PLA particles of the same size, which can be attributed to the higher porosity of dimpled particles, due to the presence of non-solvent in the dispersed phase. More porous polymer matrix makes it easier for water molecules to penetrate inside the particles, which results in faster polymer degradation and drug release.

The release rate of paracetamol in Fig. 12 is very high as a result of the high solubility of paracetamol in water $(17.4 \mathrm{~g} / \mathrm{kg})$ and high solubility of ethyl acetate in water (8.3 \%). Irrespective of the fast release profiles, the rate of drug release was precisely controlled by the microstructure of the particles.

Janus and Hemispherical Polymer Particles. Biodegradable PLA-PCL Janus particles were formed by solvent evaporation-induced internal phase separation from emulsion droplets composed of $3 \%(\mathrm{v} / \mathrm{v})$ PCL/PLA mixture (30/70 or 70/30) dissolved in DCM. Confocal microscopic images of Janus particles stained with Rh6G and Nile Red are shown in Figure 13. The fluorescence emission spectrum of $\mathrm{Rh} 6 \mathrm{G}$ is in the range from $\approx 500-700 \mathrm{~nm}$ (green to red visible range) and that of Nile red is above $550 \mathrm{~nm}$ (mainly visible red fluorescence range). Therefore, green emission captured by photomultiplier tube 1 (PMT1) at $515 \pm 30 \mathrm{~nm}$ should reveal the presence of Rh6G. When PLA and PCL films are stained with Rh6G, only PLA film exhibits green fluorescence. A strong preferential sorption of Rh6G onto PLA was successfully utilized to identify and visualize the portions of the particles with the presence of PLA. As shown in the inset image in Figures 13 (b) and (e), bright green regions occupy 
respectively $1 / 3$ and 2/3 of the total particle area, corresponding to the proportion of PLA in the polymer mixture. It shows that phase separation within the droplets was complete with only trace amounts of PLA present in PCL portions and vice versa. Nile red binds nonspecifically to both polymers, resulting in red fluorescence of entire Janus particle (Figure 13 c and f). However, PLA portions exhibit a brighter shade of red, due to contribution from both Nile red and Rh6G to the total light emission. The preferential sorption observed here for the model drug Rh6G could be utilized for controlled drug loading as proportions of constituent polymers in the formulation can be varied in a controlled fashion. Also, coencapsulation of drugs could be possible since a second drug with high affinity for PCL could be introduced to effect simultaneous controlled release of two drugs. The release pattern can be controlled by the rate of degradation of the constituent polymers. The degradation properties could be harnessed to achieve multiple site drug delivery, e.g. particles produced from combination of a low and high $\mathrm{pH}$ soluble polymer would deliver drugs to gastric and enteric regions respectively from a single dose. 

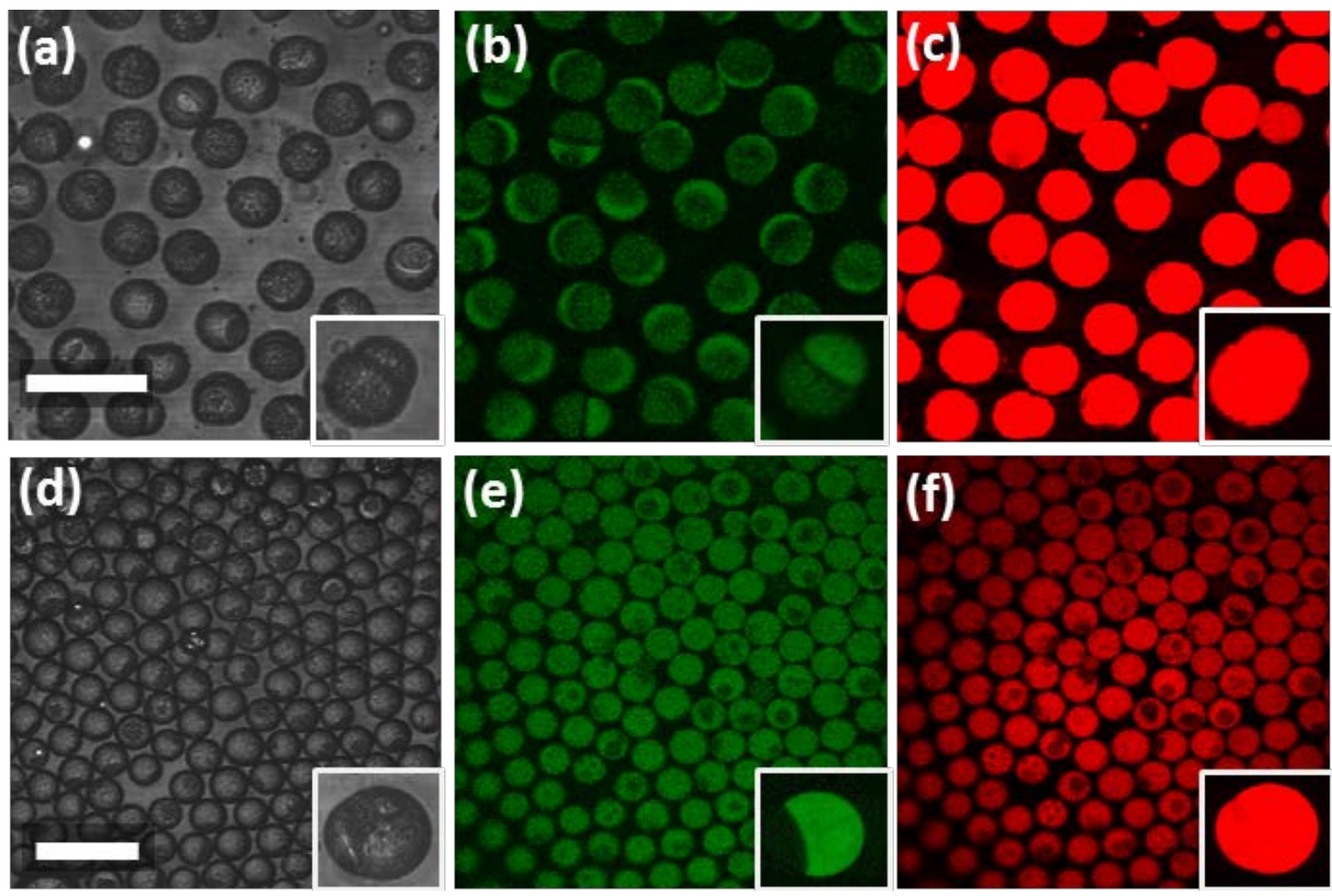

Figure 13. Confocal microscopic images of Janus PLA-PCL particles: (a)-(c) 30:70 (v/v) PLA/PCL ratio; (d)-(f) 70/30 (v/v) PLA/PCL ratio; (a) and (d) Optical images; (b) and (e) PMT1 images (emission 515 $\pm 30 \mathrm{~nm}$ ); (c) and (f) PMT2 images (emission above $570 \mathrm{~nm}$ ). Scale bar: $50 \mu \mathrm{m}$ on (a) applies for (a)-(c); $50 \mu \mathrm{m}$ on (d) applies for (e)-(f).

SEM images of monodispersed Janus particles are shown in Figure 14a. Uniform domeabsent hemispherical PCL particles formed by the dissolution of the PLA dome (i.e hemispherical cap) by acetone are shown in Figure 14b. These particles are formed because amorphous PLA is more soluble in acetone than PCL. The size of hemispherical particles can be tuned by changing the volume ratio of the solvent resistant polymer in Janus particles. Consequently, the dissolved polymer which can be recovered and reused, acts as a size and shape adjustment tool. The unique morphology of Janus and hemispherical particles can also be exploited for unique release properties when these particles are used as drug vehicles. 

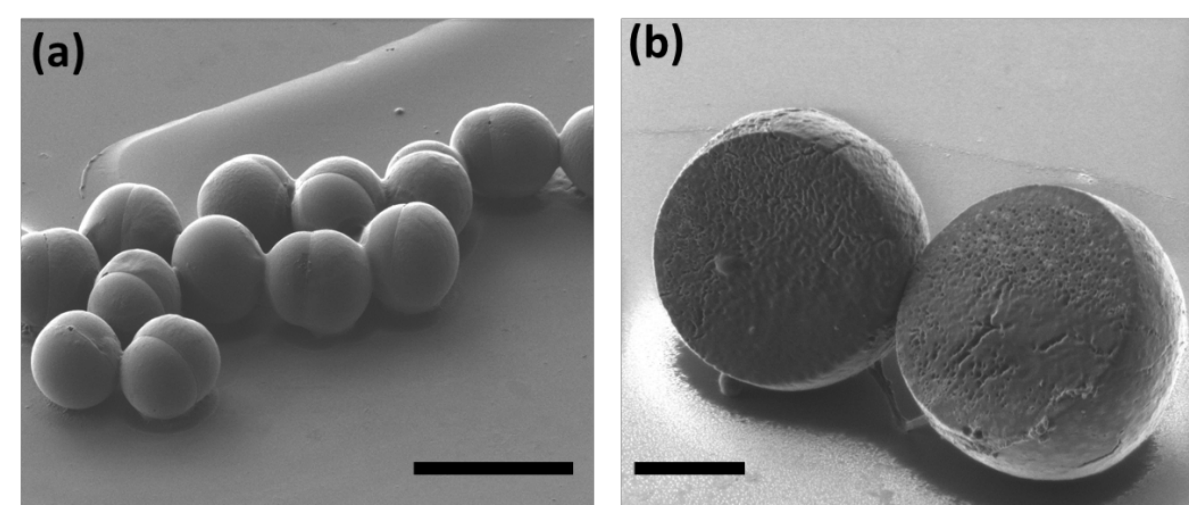

Figure 14. (a) SEM images of Janus particles with 30/70 (v/v) PLA/PCL. Dissolution of PLA dome results in hemispherical PCL particles shown in (b). Scale bar: (a) $50 \mu \mathrm{m}$ (b) $10 \mu \mathrm{m}$.

\section{CONCLUSIONS}

Synthetic polymer biodegradable microparticles with tunable size, shape, internal structure and surface roughness were produced by counter-current flow focusing in 3-D glass capillary devices. The microstructure of the particles was tailored by the addition of nanoclay, polymer non-solvent (porogen) or a second polymer in the dispersed phase prior to emulsification. The polymer matrix was found to be less porous or more porous as a result of the addition of nanoclay or 2-methylpentane, respectively, which proved to have profound effects on the release rate of paracetamol. Surface dimples which can enhance particle mobility in air and cell adhesion properties were created by adding 2-methylpentane to the dispersed phase. The size and shape of the dimples and the particle porosity were adjusted by the rate of stirring in the collection vial and the concentration of non-solvent and polymer in the dispersed phase.

Numerical modelling of fluid flow for nanoclay-loaded droplets revealed the trapping of nanoparticles in the dispersed phase at low flow rates due to vortex formation upstream of the orifice. The phenomenon of microvortex-based particle trapping can be exploited for passive separation of nanoparticles from fluid streams. 
Janus PLA/PCL particles were produced by emulsifying PLA/PCL blends (30/70 or 70/30) dissolved in dichloromethane and subsequent internal phase separation induced by solvent evaporation. A strong preferential sorption of Rhodamine 6G fluorescence dye onto PLA was successfully utilized to identify PLA portions of the Janus particles by CLSM. This could be exploited for modified drug release by polymer content variation, drug co-encapsulation and multiple site drug delivery. Uniform hemispherical PCL particles were obtained by dissolution of PLA domes with acetone. The dissolved polymer, which can be recovered and reused, acts as a size and shape adjustment tool and the increased surface area and nonspherical shape can be exploited for unique drug release properties.

Glass capillary devices were found to be extremely useful tool for preparation of polymeric microspheres with versatile morphology and structure. The particle size can easily be controlled by orifice size of collection capillary and flow rates and physical properties of both phases without orifice wetting, clogging or swelling problems.

\section{ASSOCIATED CONTENT}

Supporting Information. The supporting information includes movies of droplet generation and vortex behavior of dispersed phase flow focused in microfluidic device. It is available free of charge via the Internet at http://pubs.acs.org.

\section{ACKNOWLEDGMENT}

Ekanem E. Ekanem holds a scholarship from Niger Delta Development Commission (NDDC), Nigeria. The authors thank Dr Keith Yendall and Stephen Pickering of the Loughborough Materials and Characterisation Centre, Institute of Polymer Technology and 
Materials Engineering, Loughborough University, United Kingdom for the XRD analysis and microimaging respectively and Dr Noreen Thomas for nanoclay samples.

\section{REFERENCES}

(1) Viseras, C.; Aguzzi, C.; Cerezo, P.; Bedmar, M. C. Biopolymer-Clay Nanocomposites for Controlled Drug Delivery. Mater. Sci. Technol. 2008, 24, 1020-1026.

(2) Rodrigues, L. A. de S.; Figueiras, A.; Veiga, F.; de Freitas, R. M.; Nunes, L. C. C.; da Silva Filho, E. C.; da Silva Leite, C. M. The Systems Containing Clays and Clay Minerals from Modified Drug Release: A Review. Colloids Surf. B. 2013, 103, 642651.

(3) Martín, M. J.; Calpena, A. C.; Fernández, F.; Mallandrich, M.; Gálvez, P.; Clares, B. Development of Alginate Microspheres as Nystatin Carriers for Oral Mucosa Drug Delivery. Carbohydr. Polym. 2015, 117, 140-149.

(4) Mou, F.; Chen, C.; Zhong, Q.; Yin, Y.; Ma, H.; Guan, J. Autonomous Motion and Temperature-Controlled Drug Delivery of Mg/Pt-Poly(N-isopropylacrylamide) Janus Micromotors Driven by Simulated Body Fluid and Blood Plasma. ACS Appl. Mater. Interfaces 2014, 6, 9897-9903.

(5) Saladini, B.; Bigucci, F.; Cerchiara, T.; Gallucci, M. C.; Luppi, B. Microparticles Based on Chitosan/Pectin Polyelectrolyte Complexes for Nasal Delivery of Tacrine Hydrochloride. Drug Deliv. Transl. Res. 2013, 3, 33-41.

(6) Acosta, C.; Pérez-Esteve, E.; Fuenmayor, C. a.; Benedetti, S.; Cosio, M. S.; Soto, J.; Sancenón, F.; Mannino, S.; Barat, J.; Marcos, M. D.; Martínez-Máñez, R. Polymer composites containing gated mesoporous materials for on-command controlled release.ACS Appl. Mater. Interfaces 2014, 6, 6453-6460.

(7) Ummadi, S.; Shravani, B.; Rao, N. G. R.; Reddy, M. S.; Sanjeev, B. Overview on Controlled Release Dosage Form. Int. J. Pharma Sci. 2013, 3, 258-269.

(8) Almeida, A.; Possemiers, S.; Boone, M. N.; De Beer, T.; Quinten, T.; Van Hoorebeke, L.; Remon, J. P.; Vervaet, C. Ethylene Vinyl Acetate as Matrix for Oral Sustained Release Dosage Forms Produced via Hot-Melt Extrusion. Eur. J. Pharm. Biopharm. 2011, 77, 297-305.

(9) Xue, B.; Kozlovskaya, V.; Liu, F.; Chen, J.; Williams, J. F.; Campos-Gomez, J.; Saeed, M.; Kharlampieva, E. Intracellular Degradable Hydrogel Cubes and Spheres for AntiCancer Drug Delivery. ACS Appl. Mater. Interfaces 2015, 7, 13633-13644. 
(10) Knop, K.; Hoogenboom, R.; Fischer, D.; Schubert, U. S. Poly(ethylene Glycol) in Drug Delivery: Pros and Cons as Well as Potential Alternatives. Angew. Chem. Int. Ed. 2010, 49, 6288-6308.

(11) Kim, M. R.; Lee, S.; Park, J. K.; Cho, K. Y. Golf Ball-Shaped PLGA Microparticles with Internal Pores Fabricated by Simple O/W Emulsion. Chem. Commun. (Camb). 2010, 46, 7433-7435.

(12) Anderson, J. M.; Shive, M. S. Biodegradation and Biocompatibility of PLA and PLGA Microspheres. Adv. Drug Deliv. Rev. 2012, 64, 72-82.

(13) Vladisavljević, G. T.; Shahmohamadi, H.; Das, D. B.; Ekanem, E. E.; Tauanov, Z.; Sharma, L. Glass Capillary Microfluidics for Production of Monodispersed Poly (dlLactic Acid) and Polycaprolactone Microparticles: Experiments and Numerical Simulations. J. Colloid Interface Sci. 2014, 418, 163-170.

(14) Li, M.; Rouaud, O.; Poncelet, D. Microencapsulation by Solvent Evaporation: State of the Art for Process Engineering Approaches. Int. J. Pharm. 2008, 363, 26-39.

(15) Ha, J. U.; Xanthos, M. Drug Release Characteristics from Nanoclay Hybrids and Their Dispersions in Organic Polymers. Int. J. Pharm. 2011, 414, 321-331.

(16) Loxley, A.; Vincent, B. Preparation of Poly (methylmethacrylate) Microcapsules with Liquid Cores. J. Colloid Interface Sci. 1998, 208, 49-62.

(17) Kim, S. H.; Abbaspourrad, A.; Weitz, D. A. Amphiphilic Crescent-Moon-Shaped Microparticles Formed by Selective Adsorption of Colloids. J. Am. Chem. Soc. 2011, 133, 5516-5524.

(18) Wang, W.; Zhang, M. J.; Xie, R.; Ju, X. J.; Yang, C.; Mou, C. L.; Weitz, D. A; Chu, L. Y. Hole-Shell Microparticles from Controllably Evolved Double Emulsions. Angew. Chem. Int. Ed. 2013, 52, 8084-8087.

(19) Liu, S.; Cai, M.; Deng, R.; Wang, J.; Liang, R.; Zhu, J. Fabrication of Porous Polymer Microparticles with Tunable Pore Size and Density through the Combination of Phase Separation and Emulsion-Solvent Evaporation Approach. Korea-Australia Rheol. J. 2014, 26, 63-71.

(20) Hwangbo, K. H.; Kim, M. R.; Lee, C. S.; Cho, K. Y. Facile Fabrication of Uniform Golf-Ball-Shaped Microparticles from Various Polymers. Soft Matter 2011, 7, 10874 10878.

(21) Karbstein, H.; Schubert, H. Developments in the Continuous Mechanical Production of Oil-in-Water Macro-Emulsions. Chem. Eng. Process. 1995, 34, 205-211.

(22) Sun, G.; Qi, F.; Wu, J.; Ma, G.; Ngai, T. Preparation of uniform particle-stabilized emulsions using SPG membrane emulsification. Langmuir 2014, 30, 7052-7056.

(23) Vladisavljević, G. T.; Williams, R. A. Manufacture of Large Uniform Droplets Using Rotating Membrane Emulsification. J. Colloid Interface Sci. 2006, 299, 396-402. 
(24) Vladisavljević, G. T.; Wang, B.; Dragosavac, M. M.; Holdich, R. G. Production of Food-Grade Multiple Emulsions with High Encapsulation Yield Using Oscillating Membrane Emulsification. Colloids Surf., A 2014, 458, 78-84.

(25) Sugiura, S.; Nakajima, M.; Seki, M. Prediction of Droplet Diameter for Microchannel Emulsification. Langmuir 2002, 18, 3854-3859.

(26) Keohane, K.; Brennan, D.; Galvin, P.; Griffin, B. T. Silicon Microfluidic Flow Focusing Devices for the Production of Size- Controlled PLGA Based Drug Loaded Microparticles. Int J. Pharm. 2014, 467, 60-69.

(27) Deng, N. N.; Wang, W.; Ju, X. J.; Xie, R.; Weitz, D. A; Chu, L. Y. Wetting-Induced Formation of Controllable Monodisperse Multiple Emulsions in Microfluidics. Lab Chip 2013, 13, 4047-4052.

(28) Shum, H. C.; Abate, A. R.; Lee, D.; Studart, A. R.; Wang, B.; Chen, C. H.; Thiele, J.; Shah, R. K.; Krummel, A.; Weitz, D. A. Droplet Microfluidics for Fabrication of NonSpherical Particles. Macromol. Rapid Commun. 2010, 31, 108-118.

(29) Seemann, R.; Brinkmann, M.; Pfohl, T.; Herminghaus, S. Droplet Based Microfluidics. Reports Prog. Phys. 2012, 75, 16601.

(30) Vladisavljević, G. T.; Khalid, N.; Neves, M. A.; Kuroiwa, T.; Nakajima, M.; Uemura, K.; Ichikawa, S.; Kobayashi, I. Industrial Lab-on-a-Chip: Design, Applications and Scale-up for Drug Discovery and Delivery. Adv. Drug Deliv. Rev. 2013, 65, 16261663.

(31) Vladisavljević, G. T.; Kobayashi, I.; Nakajima, M. Generation of Highly Uniform Droplets Using Asymmetric Microchannels Fabricated on a Single Crystal Silicon Plate: Effect of Emulsifier and Oil Types. Powder Technol. 2008, 183, 37-45.

(32) Utada, A S.; Lorenceau, E.; Link, D. R.; Kaplan, P. D.; Stone, H. a; Weitz, D. a. Monodisperse Double Emulsions Generated from a Microcapillary Device. Science 2005, 308, 537-541.

(33) Utada, A. S.; Chu, L.-Y.; Fernandez-Nieves, A.; Link, D. R.; Holtze, C.; Weitz, D. A. Dripping, Jetting, Drops, and Wetting: The Magic of Microfluidics. Mrs Bull. 2007, 32, 702-708.

(34) Lee, D.; Weitz, D. A. Nonspherical Colloidosomes with Multiple Compartments from Double Emulsions. Small 2009, 5, 1932-1935.

(35) Shum, H. C.; Kim, J.; Weitz, D. A. Microfluidic Fabrication of Monodisperse Biocompatible and Biodegradable Polymersomes with Controlled Permeability. J. Am. Chem. Soc. 2008, 10, 9543-9549.

(36) Sauret, A.; Shum, H. C. Forced Generation of Simple and Double Emulsions in AllAqueous Systems. Appl. Phys. Lett. 2012, 100, 154106. 
(37) Comunian, T. A.; Abbaspourrad, A.; Favaro-Trindade, C. S.; Weitz, D. A. Fabrication of Solid Lipid Microcapsules Containing Ascorbic Acid Using a Microfluidic Technique. Food Chem. 2014, 152, 271-275.

(38) Granberg, R. A.; Rasmuson, Å. C. Solubility of Paracetamol in Pure Solvents. J. Chem. Eng. Data 1999, 44, 1391-1395.

(39) Hirt, C. W.; Nichols, B. D. Volume of Fluid (VOF) Method for the Dynamics of Free Boundaries. J. Comput. Phys. 1981, 39, 201-225.

(40) Brackbill, J. U.; Kothe, D. B.; Zemach, C. A Continuum Method for Modeling Surface Tension. J. Comput. Phys. 1992, 100, 335-354.

(41) Vladisavljević, G. T.; Duncanson, W. J.; Shum, H. C.; Weitz, D. A. Emulsion Templating of Poly(lactic Acid) Particles: Droplet Formation Behavior. Langmuir 2012, 28, 12948-12954.

(42) Nabavi, S. A.; Gu, S.; Vladisavljević, G. T.; Ekanem, E. E. Dynamics of Double Emulsion Break-up in Three Phase Glass Capillary Microfluidic Devices. J. Colloid Interface Sci. 2015, 450, 279-287.

(43) Nabavi, S. A.; Vladisavljević, G. T.; Gu, S.; Ekanem, E. E. Double Emulsion Production in Glass Capillary Microfluidic Device: Parametric Investigation of Droplet Generation Behaviour. Chem. Eng. Sci. 2015, 130, 183-196.

(44) ANSYS® Fluent 15.0. Users’ guide. ANSYS Inc.; 2013.

(45) Di Carlo, D. Inertial Microfluidics. Lab Chip 2009, 9, 3038-3046.

(46) Sollier, E.; Go, D. E.; Che, J.; Gossett, D. R.; O’Byrne, S.; Weaver, W. M.; Kummer, N.; Rettig, M.; Goldman, J.; Nickols, N.; McCloskey, S.; Kulkarni, R. P.; Di Carlo, D. Size-Selective Collection of Circulating Tumor Cells Using Vortex Technology. Lab Chip 2014, 14, 63-77.

(47) Akers, M.J. Parenteral Quality Control: Sterility, Pyrogen Particulate and Package Integrity Testing. $2^{\text {nd }}$ Ed, New York: Marcel Dekker 1994, 179.

(48) Veldhuis, G.; Gironés, M.; Bingham, D. Monodisperse Microspheres for Parenteral Drug Delivery. Drug Deliv. Technol. 2009, 9, 23-31.

(49) Wischke, C.; Schwendeman, S. P. Principles of Encapsulating Hydrophobic Drugs in PLA/PLGA Microparticles. Int. J. Pharm. 2008, 364, 298-327.

(50) Norrish, K. The Swelling of Montmorillonite. Discuss. Faraday Soc. 1954, 18, 120-134.

(51) Metz, S.; Anderson, R. L.; Geatches, D. L.; Suter, J. L.; Lines, R.; Greenwell, H. C. Understanding the Swelling Behavior of Modified Nanoclay Filler Particles in Water and Ethanol. J. Phys. Chem. C 2015, 119, 12625-12642. 
(52) Duan, Z.; Thomas, N. L.; Huang, W. Water Vapour Permeability of Poly(lactic Acid) Nanocomposites. J. Memb. Sci. 2013, 445, 112-118.

(53) Lee, J. H.; Lee, C. S.; Cho, K. Y. Enhanced Cell Adhesion to the Dimpled Surfaces of Golf-Ball-Shaped Microparticles. ACS Appl. Mater. Interfaces 2014, 6, 16493-16497.

(54) Donovan, M. J.; Smyth, H. D. C. Influence of Size and Surface Roughness of Large Lactose Carrier Particles in Dry Powder Inhaler Formulations. Int. J. Pharm. 2010, 402, 1-9. 
Table of Contents Graphic

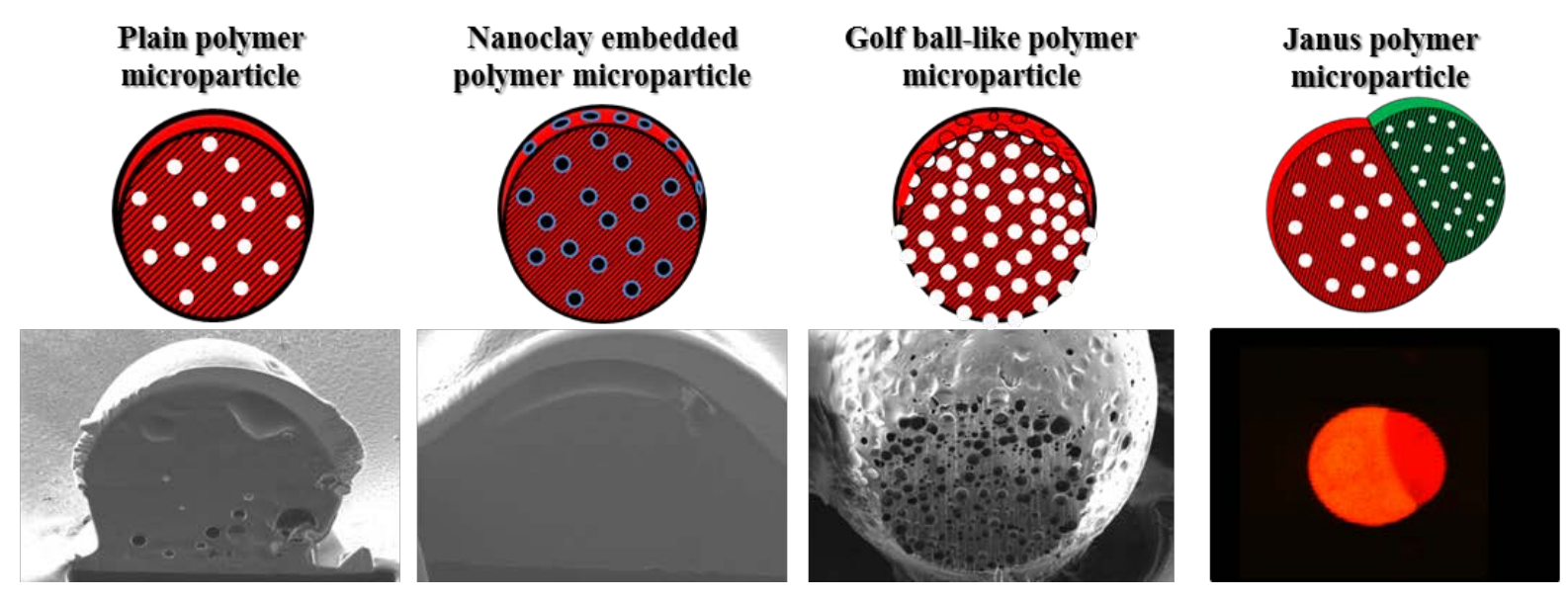

